\title{
SPLIT OBJECTS WITH RESPECT TO A FULLY INVARIANT SHORT EXACT SEQUENCE IN ABELIAN CATEGORIES
}

\author{
SEPTIMIU CRIVEI, DERYA KESKIN TÜTÜNCÜ, AND RACHID TRIBAK \\ Dedicated to the memory of Professor John Clark
}

\begin{abstract}
We introduce and investigate (dual) relative split objects with respect to a fully invariant short exact sequence in abelian categories. We compare them with (dual) relative Rickart objects, and we study their behaviour with respect to direct sums and classes all of whose objects are (dual) relative split. We also introduce and study (dual) strongly relative split objects. Applications are given to Grothendieck categories, module and comodule categories.
\end{abstract}

\section{INTRODUCTION}

Right Rickart rings have the root in the work of Rickart [30 on certain Banach algebras. In an arbitrary ring-theoretic setting they were considered by Maeda 25, who defined them as rings in which the right annihilator of any element is generated by an idempotent. Independently, Hattori [18] defined right p.p. rings as rings in which every principal right ideal is projective. Later on, it turned out that the two concepts are actually equivalent, and their theory was further developed by many mathematicians. Some classical examples of right Rickart rings include right semihereditary rings, Baer rings and von Neumann regular rings.

A natural development of the theory of Rickart rings was towards module theory. Thus, Lee, Rizvi and Roman introduced and extensively studied Rickart modules and dual Rickart modules in a series of papers [22, 23, 24], which extended the theory of Baer modules [31] and dual Baer modules [21]. A further generalization was considered by Crivei and Kör [5], who investigated relative Rickart objects and dual relative Rickart objects in abelian categories (also, see [6, 7]). If $M$ and $N$ are objects of an abelian category, then $N$ is called $M$-Rickart if for every morphism $f: M \rightarrow N, \operatorname{ker}(f)$ is a section, while $N$ is called dual $M$-Rickart if for every morphism $f: M \rightarrow N$, coker $(f)$ is a retraction. Their motivation was to set a unified theory of relative Rickart objects with versatile applications, which allows one to deduce naturally properties of dual relative Rickart objects (by the duality principle), relative regular objects (which are relative Rickart and dual relative Rickart) in the sense of Dăscălescu, Năstăsescu, Tudorache and Dăuş [11, 12] as well as relative Baer objects (as particular relative Rickart objects) and dual relative Baer objects (by the duality principle) [5, 6, 7]. In recent years, Rickart modules and dual Rickart modules were generalized to (dual) $t$-Rickart modules by Asgari and Haghany [1], (dual) T-Rickart modules by Ebrahimi Atani, Khoramdel and Dolati Pish Hesari [14, 16] and (dual) $F$-inverse split modules by Ungor, Halicioglu and Harmanci [35, 36], the latter developing a theory using arbitrary fully invariant submodules.

In the present paper we study (dual) relative split objects with respect to a fully invariant short exact sequence in abelian categories. Note that any preradical $r$ of a category gives rise to a fully invariant short exact sequence $0 \rightarrow r(M) \rightarrow M \rightarrow M / r(M) \rightarrow 0$ for every object $M$. If $M$ is an object and $0 \rightarrow F \stackrel{i}{\rightarrow} N \stackrel{d}{\rightarrow} C \rightarrow 0$ is a fully invariant short exact sequence, then $N$ is $M$-F-split if and only if for every morphism $g: M \rightarrow N$, $\operatorname{ker}(d g)$ is a section, while $N$ is dual $M$ $F$-split if and only if for every morphism $g: N \rightarrow M$, coker $(g i)$ is a retraction. For $M=N$, one has the notion of (dual) self- $F$-split object. We also introduce a strong version of (dual) relative split object, which generalizes (dual) strong Rickartness in the sense of [7, 15, 39]. Relative split

Date: March 13, 2018.

2010 Mathematics Subject Classification. 18E10, 18E15, 16D90.

Key words and phrases. Abelian category, fully invariant short exact sequence, (dual) (strongly) $F$-split object, (dual) (strongly) Rickart object. 
objects generalize all the above Rickart-type modules as well as extending modules. Note that $N$ is $M$-Rickart if and only if $N$ is $M$-0-split, while $N$ is dual $M$-Rickart if and only if $N$ is dual $M$ - $M$-split. Moreover, $M$ is (dual) self- $F$-split if and only if $M \cong F \oplus C$ and $C$ is self-Rickart ( $F$ is dual self-Rickart). When $F=r(M)$ for some (pre)radical $r$ of $\mathcal{A}$, this shows that self- $F$-split and dual self- $F$-split objects are some particular objects for which their (pre)torsion part splits off (see the splitting problem discussed by Chase [3], Goodearl [17], Kaplansky [19], Năstăsescu and Torrecillas [27] or Teply [34]). Applications are given to Grothendieck categories, module and comodule categories.

Our results will usually have two parts, out of which we only prove the first one, the second one following by the duality principle in abelian categories. In Section 2 we present some needed terminology and properties on fully invariant short exact sequences in abelian categories. In Section 3 we introduce (strongly) relative split objects with respect to a fully invariant short exact sequence $0 \rightarrow F \rightarrow N \rightarrow C \rightarrow 0$ in an abelian category $\mathcal{A}$. We show that an object $M$ of $\mathcal{A}$ is strongly self- $F$-split if and only if $M$ is self- $F$-split and every direct summand of $M$ which contains $F$ is fully invariant. We prove that every (strongly) self- $F$-split object has the summand intersection property for (fully invariant) direct summands containing $F$. One of our main results shows that if $e: M \rightarrow M^{\prime}$ is an epimorphism, $m: N^{\prime} \rightarrow N$ is a monomorphism, the inclusion monomorphism $u: F \cap N^{\prime} \rightarrow N^{\prime}$ is fully invariant and $N$ is (strongly) $M-F$-split,

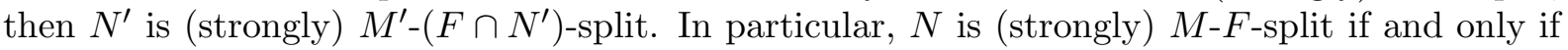
for every direct summand $M_{1}$ of $M$ and for every direct summand $N_{1}$ of $N, N_{1}$ is (strongly) $M_{1}$ - $\left(F \cap N_{1}\right)$-split. In Section 4 we compare relative self- $F$-split objects and relative self-Rickart objects. We prove that $M$ is (strongly) self- $F$-split if and only if $M \cong F \oplus C$ and $C$ is (strongly) self-Rickart. We also show that $M$ is strongly self- $F$-split if and only if $M$ is self- $F$-split and the ring $\operatorname{End}_{\mathcal{A}}(C)$ is abelian. In Section 5 we deal with coproducts of relative split objects. If $M$ and $N$ are objects of $\mathcal{A}, N=\bigoplus_{k=1}^{n} N_{k}$ is a direct sum decomposition, and $0 \rightarrow F \rightarrow N \rightarrow C \rightarrow 0$ is a fully invariant short exact sequence in $\mathcal{A}$, then it is shown that $N$ is (strongly) $M-F$ split if and only if $N_{k}$ is (strongly) $M-\left(F \cap N_{k}\right)$-split for every $k \in\{1, \ldots, n\}$. Also, if $r$ is a preradical of $\mathcal{A}, M$ is an object of $\mathcal{A}$ having the strong summand intersection property for (fully invariant) direct summands containing $r(M)$ and $\left(N_{k}\right)_{k \in K}$ is a family of objects of $\mathcal{A}$, then $\bigoplus_{k \in K} N_{k}$ is (strongly) $M-r\left(\bigoplus_{k \in K} N_{k}\right)$-split if and only if $N_{k}$ is (strongly) $M-r\left(N_{k}\right)$-split for every $k \in K$. In Section 6 we study classes all of whose objects are self- $F$-split. We characterize spectral categories, locally finitely generated Grothendieck categories which are $V$-categories or regular categories, (semi)hereditary categories with enough projectives and co(semi)hereditary categories with enough injectives in terms of self- $F$-splitness or dual self- $F$-splitness of objects of certain classes. If an abelian category $\mathcal{A}$ has a generator $G$ and enough injectives, and $r$ is a radical of $\mathcal{A}$, then we characterize when $G / r(G)$ is (semi)hereditary. Also, if $\mathcal{A}$ has an injective cogenerator $G$ and $r$ is a preradical of $\mathcal{A}$, then we establish equivalent conditions for $G$ to be (strongly) self- $r(G)$-split. Finally, in Section 7 we give some further applications to module and comodule categories. Among them, we show that every (strongly) extending module $N$ is (strongly) self- $F$-split, where $F$ is the second singular submodule of $N$.

Let us finally note that the transfer via functors between abelian categories of the (dual) relative splitness of objects with respect to a fully invariant short exact sequence is studied in the separate paper [4].

\section{Fully invariant Short ExaCt SEQUences}

Let $\mathcal{A}$ be an abelian category. For every morphism $f: M \rightarrow N$ in $\mathcal{A}$ we denote its kernel, cokernel, coimage and image by $\operatorname{ker}(f): \operatorname{Ker}(f) \rightarrow M, \operatorname{coker}(f): N \rightarrow \operatorname{Coker}(f), \operatorname{coim}(f)$ : $M \rightarrow \operatorname{Coim}(f)$ and $\operatorname{im}(f): \operatorname{Im}(f) \rightarrow N$ respectively. Since $\mathcal{A}$ is an abelian category, one has $\operatorname{Coim}(f) \cong \operatorname{Im}(f)$. For a short exact sequence $0 \rightarrow A \rightarrow B \rightarrow C \rightarrow 0$ in $\mathcal{A}$, we sometimes write $C=B / A$. Recall that a morphism $f: M \rightarrow N$ is called a section (retraction) if there exists a morphism $f^{\prime}: N \rightarrow M$ such that $f^{\prime} f=1_{M}\left(f f^{\prime}=1_{N}\right)$.

Now we recall some terminology on fully invariant kernels from [7], and we establish a series of properties, which generalize corresponding properties of fully invariant submodules of modules, and will be needed in the next sections of the paper. 
Definition 2.1. [7, Definition 2.2] Let $\mathcal{A}$ be an abelian category.

(1) A kernel (monomorphism) $i: K \rightarrow M$ in $\mathcal{A}$ is called fully invariant if for every morphism $h: M \rightarrow M, h i$ factors through $i$.

(2) A cokernel (epimorphism) $d: M \rightarrow C$ in $\mathcal{A}$ is called fully coinvariant if for every morphism $h: M \rightarrow M, d h$ factors through $d$.

Lemma 2.2. [7, Lemma 2.5] Let $\mathcal{A}$ be an abelian category. Then a kernel $i: K \rightarrow M$ in $\mathcal{A}$ is fully invariant if and only if its cokernel $d: M \rightarrow C$ is fully coinvariant.

Definition 2.3. Let $\mathcal{A}$ be an abelian category. A short exact sequence $0 \rightarrow A \stackrel{i}{\rightarrow} B \stackrel{d}{\rightarrow} C \rightarrow 0$ in $\mathcal{A}$ is called fully invariant if $i$ is fully invariant, or equivalently, $d$ is fully coinvariant.

Example 2.4. [7, Example 2.3] Consider the category $\operatorname{Mod}(R)$ of unitary right modules over a ring $R$ with identity. Then a kernel $i: K \rightarrow M$ is fully invariant if and only if for every endomorphism $h: M \rightarrow M, h i=i \alpha$ for some homomorphism $\alpha: K \rightarrow K$ if and only if for every endomorphism $h: M \rightarrow M, h(K)=\operatorname{Im}(h i) \subseteq \operatorname{Im}(i)=K$ if and only if $K$ is a fully invariant submodule of $M$.

Lemma 2.5. [7, Lemma 2.4] Let $\mathcal{A}$ be an abelian category.

(1) The composition of two fully invariant kernels in $\mathcal{A}$ is a fully invariant kernel.

(2) The composition of two fully coinvariant cokernels in $\mathcal{A}$ is a fully coinvariant cokernel.

Proposition 2.6. Let $\mathcal{A}$ be an abelian category. Let $0 \rightarrow F \stackrel{i}{\rightarrow} M \stackrel{d}{\rightarrow} M / F \rightarrow 0$ be a fully invariant short exact sequence in $\mathcal{A}$ and $M=M_{1} \oplus M_{2}$. Then:

(1) $F \cong\left(F \cap M_{1}\right) \oplus\left(F \cap M_{2}\right)$.

(2) $M / F \cong M /\left(F+M_{1}\right) \oplus M /\left(F+M_{2}\right)$.

Proof. (1) For $l=1,2$, denote by $u_{l}: M_{l} \rightarrow M$ the canonical injection, and by $p_{l}: M \rightarrow M_{l}$ the canonical projection. Also, for $l=1,2$, denote by $j_{l}: F \cap M_{l} \rightarrow M_{l}$ and $k_{l}: F \cap M_{l} \rightarrow F$ the inclusion monomorphisms. The universal property of the coproduct yields the monomorphism $\left[\begin{array}{ll}k_{1} & k_{2}\end{array}\right]:\left(F \cap M_{1}\right) \oplus\left(F \cap M_{2}\right) \rightarrow F$.

Since $i: F \rightarrow M$ is fully invariant, there exist morphisms $\alpha_{1}, \alpha_{2}: F \rightarrow F$ such that $u_{1} p_{1} i=i \alpha_{1}$ and $u_{2} p_{2} i=i \alpha_{2}$. Then $i\left(\alpha_{1}+\alpha_{2}\right)=u_{1} p_{1} i+u_{2} p_{2} i=i$, hence $\alpha_{1}+\alpha_{2}=1_{F}$, because $i$ is a monomorphism. For $l=1,2$ the following square is a pullback:

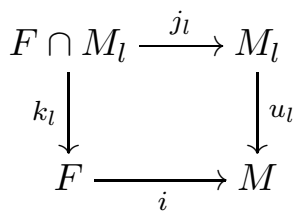

For $l=1,2$, by the pullback property, there exists a morphism $\gamma_{l}: F \rightarrow F \cap M_{l}$ such that $j_{l} \gamma_{l}=i_{l}$ and $k_{l} \gamma_{l}=\alpha_{l}$. Then $\left[k_{1} k_{2}\right]\left[\begin{array}{l}\gamma_{1} \\ \gamma_{2}\end{array}\right]=k_{1} \gamma_{1}+k_{2} \gamma_{2}=\alpha_{1}+\alpha_{2}=1_{F}$. Hence $\left[k_{1} k_{2}\right]$ is a retraction, and so it is an isomorphism. This shows that $F \cong\left(F \cap M_{1}\right) \oplus\left(F \cap M_{2}\right)$.

Proposition 2.7. Let $\mathcal{A}$ be an abelian category. Let $0 \rightarrow F \stackrel{i}{\rightarrow} M \stackrel{d}{\rightarrow} M / F \rightarrow 0$ be a fully invariant short exact sequence and $0 \rightarrow G \stackrel{j}{\rightarrow} M \stackrel{p}{\rightarrow} M / G \rightarrow 0$ a short exact sequence in $\mathcal{A}$.

(1) Let $u: F \cap G \rightarrow G$ be the inclusion monomorphism.

(i) Assume that the above second short exact sequence is also fully invariant. Then the inclusion monomorphism $j u: F \cap G \rightarrow M$ is fully invariant.

(ii) Assume that every morphism $G \rightarrow G$ can be extended to a morphism $M \rightarrow M$ in $\mathcal{A}$. Then $u: F \cap G \rightarrow G$ is fully invariant.

(2) Let $q: M / G \rightarrow M /(F+G)$ be the induced epimorphism.

(i) Assume that the above second short exact sequence is also fully invariant. Then the induced epimorphism $q p: M \rightarrow M /(F+G)$ is fully coinvariant.

(ii) Assume that every morphism $M / G \rightarrow M / G$ can be lifted to a morphism $M \rightarrow M$ in $\mathcal{A}$. Then $q: M / G \rightarrow M /(F+G)$ is fully coinvariant. 
Proof. (1) Denote by $k: F \cap G \rightarrow F$ the inclusion monomorphism.

(i) Let $f: M \rightarrow M$ be a morphism in $\mathcal{A}$. Since $i$ and $j$ are fully invariant kernels, there exist morphisms $\alpha: F \rightarrow F$ and $\beta: G \rightarrow G$ such that $i \alpha=f i$ and $j \beta=f j$. The following commutative square is a pullback:

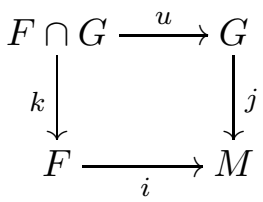

We have $i \alpha k=f i k=f j u=j \beta u$. By the pullback property, there exists a unique morphism $\gamma: F \cap G \rightarrow F \cap G$ such that $k \gamma=\alpha k$ and $u \gamma=\beta u$. We have $f j u=j \beta u=j u \gamma$. Hence $j u: F \cap G \rightarrow M$ is fully invariant.

(ii) Let $h: G \rightarrow G$ be a morphism in $\mathcal{A}$. By hypothesis, $h$ can be extended to a morphism $f: M \rightarrow M$. Hence $f j=j h$. Since $i$ is fully invariant, there exists a morphism $\alpha: F \rightarrow F$ such that $i \alpha=f i$. Consider the pullback square from the proof of (i). We have $i \alpha k=f i k=f j u=$ $j h u$. By the pullback property, there exists a unique morphism $\gamma: F \cap G \rightarrow F \cap G$ such that $k \gamma=\alpha k$ and $u \gamma=h u$. This shows that $u$ is fully invariant.

Corollary 2.8. Let $\mathcal{A}$ be an abelian category. Let $0 \rightarrow F \rightarrow M \rightarrow M / F \rightarrow 0$ be a fully invariant short exact sequence in $\mathcal{A}$ and $M=M_{1} \oplus M_{2}$. Then:

(1) The inclusion monomorphism $u: F \cap M_{1} \rightarrow M_{1}$ is fully invariant.

(2) The induced epimorphism $q: M_{1} \rightarrow M /\left(F+M_{2}\right)$ is fully coinvariant.

Proof. (1) Note that every morphism $M_{1} \rightarrow M_{1}$ can be extended to a morphism $M \rightarrow M$ in $\mathcal{A}$, and use Proposition 2.7 (1) (ii).

Proposition 2.9. Let $\mathcal{A}$ be an abelian category.

(1) Let $g: B \rightarrow B^{\prime}$ be a cokernel and $d^{\prime}: B^{\prime} \rightarrow C$ a morphism such that $d=d^{\prime} g: B \rightarrow C$ is a fully coinvariant retraction. Then $d^{\prime}$ is a fully coinvariant retraction.

(2) Let $g: B^{\prime} \rightarrow B$ be a kernel and $i^{\prime}: A \rightarrow B^{\prime}$ a morphism such that $i=g i^{\prime}: A \rightarrow B$ is a fully invariant section. Then $i^{\prime}$ is a fully invariant section.

Proof. (1) Note that $d^{\prime}$ is a retraction. One may construct the following commutative diagram:

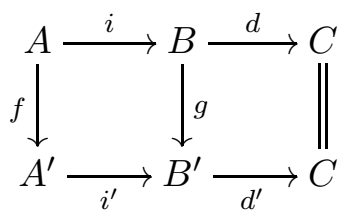

where the rows are exact and the left square is a pullback. Then $f$ is a cokernel and $i$ is a fully invariant section. Now let $h: B^{\prime} \rightarrow B^{\prime}$ be a morphism. There exists a monomorphism $s: C \rightarrow B$ such that $d s=1_{C}$. Consider the morphism $s d^{\prime} h g: B \rightarrow B$. Since $i$ is a fully invariant section, there exists a morphism $\alpha: A \rightarrow A$ such that $s d^{\prime} h g i=i \alpha$. Then $d^{\prime} h i^{\prime} f=d^{\prime} h g i=d s d^{\prime} h g i=$ $d i \alpha=0$, whence we have $d^{\prime} h i^{\prime}=0$, because $f$ is an epimorphism. Then there exists a morphism $\gamma: C \rightarrow C$ such that $d^{\prime} h=\gamma d^{\prime}$. Hence $d^{\prime}$ is a fully coinvariant retraction.

Proposition 2.10. Let $\mathcal{A}$ be an abelian category.

(1) Let $\left[\begin{array}{cc}i & 0 \\ 0 & i^{\prime}\end{array}\right]: A \oplus A^{\prime} \rightarrow B \oplus B^{\prime}$ be a fully invariant kernel in $\mathcal{A}$. Then $i: A \rightarrow B$ and $i^{\prime}: A^{\prime} \rightarrow B^{\prime}$ are fully invariant kernels.

(2) Let $\left[\begin{array}{ll}d & 0 \\ 0 & d^{\prime}\end{array}\right]: B \oplus B^{\prime} \rightarrow C \oplus C^{\prime}$ be a fully coinvariant cokernel in $\mathcal{A}$. Then $d: B \rightarrow C$ and $d^{\prime}: B^{\prime} \rightarrow C^{\prime}$ are fully coinvariant cokernels.

Proof. (1) Clearly, $i$ is a kernel. Let $h: B \rightarrow B$ be a morphism. For the morphism $\left[\begin{array}{ll}h & 0 \\ 0 & 1\end{array}\right]$ : $B \oplus B^{\prime} \rightarrow B \oplus B^{\prime}$ there exists a morphism $\left[\begin{array}{ll}a & b \\ c & d\end{array}\right]: A \oplus A^{\prime} \rightarrow A \oplus A^{\prime}$ such that

$$
\left[\begin{array}{ll}
h & 0 \\
0 & 1
\end{array}\right]\left[\begin{array}{ll}
i & 0 \\
0 & i^{\prime}
\end{array}\right]=\left[\begin{array}{ll}
i & 0 \\
0 & i^{\prime}
\end{array}\right]\left[\begin{array}{ll}
a & b \\
c & d
\end{array}\right] \text {. }
$$

Then $h i=i a$, which shows that $i$ is fully invariant. 
Lemma 2.11. Let $\mathcal{A}$ be an abelian category with coproducts. Let $\left(M_{k}\right)_{k \in K}$ be a family of objects of $\mathcal{A}$ such that $\operatorname{Hom}_{\mathcal{A}}\left(M_{k}, M_{l}\right)=0$ for every $k, l \in K$ with $k \neq l$. Then the short exact sequences $0 \rightarrow F_{k} \rightarrow M_{k} \rightarrow C_{k} \rightarrow 0$ are fully invariant for every $k \in K$ if and only if the induced short exact sequence $0 \rightarrow \bigoplus_{k \in K} F_{k} \rightarrow \bigoplus_{k \in K} M_{k} \rightarrow \bigoplus_{k \in K} C_{k} \rightarrow 0$ is fully invariant.

Proof. Straightforward.

Let $\mathcal{A}$ be an abelian category. Recall that a preradical $r$ of $\mathcal{A}$ is a subfunctor of the identity functor on $\mathcal{A}$, that is, $r: \mathcal{A} \rightarrow \mathcal{A}$ is a functor which assigns to each object $A$ of $\mathcal{A}$ a subobject $r(A)$ such that every morphism $A \rightarrow B$ induces a morphism $r(A) \rightarrow r(B)$ by restriction (e.g., see [2, I.1]).

The following proposition relates fully invariant short exact sequences and preradicals, and will be implicitly used, without further reference. It is an immediate generalization of [2, Proposition I.6.2] from module categories to abelian categories.

Proposition 2.12. Let $\mathcal{A}$ be an abelian category. Then a short exact sequence $0 \rightarrow F \rightarrow M \rightarrow$ $C \rightarrow 0$ in $\mathcal{A}$ is fully invariant if and only if there is a preradical $r$ of $\mathcal{A}$ such that $r(M)=F$.

\section{3. $F$-SPLIT OBJECTS}

In this section we introduce our main concepts of (strongly) relative $F$-split and dual (strongly) relative $F$-split objects in abelian categories.

Definition 3.1. Let $\mathcal{A}$ be an abelian category. Let $M$ be an object of $\mathcal{A}$, and let $0 \rightarrow F \stackrel{i}{\rightarrow}$ $N \stackrel{d}{\rightarrow} C \rightarrow 0$ be a fully invariant short exact sequence in $\mathcal{A}$. Then $N$ is called:

(1) (strongly) $M$-F-split if for every morphism $g: M \rightarrow N$ the morphism $j: P \rightarrow M$ from the following pullback square is a (fully invariant) section:

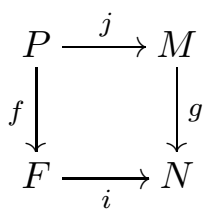

(2) dual (strongly) $M$-F-split if for every morphism $g: N \rightarrow M$ the morphism $p: M \rightarrow Q$ from the following pushout square is a (fully coinvariant) retraction:

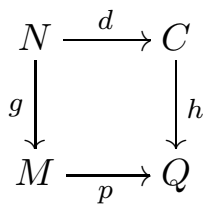

(3) (strongly) self-F-split if $N$ is (strongly) $N$-F-split.

(4) dual (strongly) self-F-split if $N$ is dual (strongly) $N$-F-split.

Lemma 3.2. Let $\mathcal{A}$ be an abelian category. Let $M$ be an object of $\mathcal{A}$, and let $0 \rightarrow F \stackrel{i}{\rightarrow} N \stackrel{d}{\rightarrow}$ $C \rightarrow 0$ be a fully invariant short exact sequence in $\mathcal{A}$. Then:

(1) $N$ is (strongly) $M-F$-split if and only if for every morphism $g: M \rightarrow N$, $\operatorname{ker}(d g)$ is a (fully invariant) section.

(2) $N$ is dual (strongly) $M$-F-split if and only if for every morphism $g: N \rightarrow M$, coker $(g i)$ is a (fully coinvariant) retraction.

Proof. (1) Consider the following diagram:

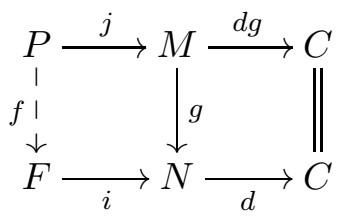


Since $i=\operatorname{ker}(d)$, the diagram may be completed to a pullback square $P M F N$ if and only if $j=\operatorname{ker}(d g)$ [26, Proposition 13.2]. Now the conclusion is clear.

Remark 3.3. Consider the module category $\operatorname{Mod}(R)$, and let $M, N$ be right $R$-modules. We refer to notation and diagrams from Definition 3.1 .

(1) Let $F$ be a fully invariant submodule of $N$. Then $N$ is (strongly) $M$ - $F$-split if and only if for every homomorphism $g: M \rightarrow N, j: P \rightarrow M$ is a (fully invariant) section if and only if for every homomorphism $g: M \rightarrow N, P=g^{-1}(F)$ is a (fully invariant) direct summand of $M$.

(2) Let $F$ be a fully invariant submodule of $M$, or equivalently, let $C=M / F$ be a fully coinvariant factor module of $M$. Then $N$ is dual (strongly) $M-F$-split if and only if for every homomorphism $g: N \rightarrow M, p: M \rightarrow Q$ is a (fully coinvariant) retraction if and only if for every homomorphism $g: N \rightarrow M, \operatorname{ker}(p)$ is a (fully invariant) section if and only if for every homomorphism $g: N \rightarrow M, g i$ is a (fully invariant) section if and only if for every homomorphism $g: N \rightarrow M, g(F)$ is a (fully invariant) direct summand of $M$.

Example 3.4. (1) Let $M$ and $N$ be objects of an abelian category $\mathcal{A}$. Obviously, $N$ is strongly $M-N$-split and $N$ is dual strongly $M$-0-split.

(2) Consider the module category $\operatorname{Mod}(R)$. Let $M$ and $N$ be right $R$-modules. Then $N$ is $M$-F-split if and only if $N$ is $M-F$-inverse split in the sense of [35]. For $F=Z_{M}^{2}(N)$ (see the notation from the last section of our paper), a module $N$ is $M$-F-split if and only if $N$ is $M$-T-Rickart in the sense of [14].

Let us note that our categorical dual notion of dual (relative) $F$-splitness does not coincide with dual (relative) $F$-inverse splitness in the sense of [36] (e.g., apart from their definitions, compare our forthcoming Theorem 4.3 and [36, Theorem 2.2]). But for $F=\bar{Z}_{M}^{2}(N)$ (see the notation from the last section of our paper), a module $N$ is dual $M$-F-split if and only if $N$ is $M-T$-dual Rickart in the sense of [16].

Strong self- $F$-splitness and self- $F$-splitness are related by the following result.

Theorem 3.5. Let $\mathcal{A}$ be an abelian category. Let $0 \rightarrow F \stackrel{i}{\rightarrow} M \stackrel{d}{\rightarrow} C \rightarrow 0$ be a fully invariant short exact sequence in $\mathcal{A}$.

(1) The following are equivalent:

(i) $M$ is strongly self-F-split.

(ii) $M$ is self-F-split and every direct summand of $M$ which contains $F$ is fully invariant.

(2) The following are equivalent:

(i) $M$ is dual strongly self-F-split.

(ii) $M$ is dual self-F-split and every direct summand of $M$ which is contained in $F$ is fully invariant.

Proof. (1) (i) $\Rightarrow$ (ii) Assume that $M$ is strongly self- $F$-split. Clearly, $M$ is self- $F$-split. Now let $X$ be a direct summand of $M$ such that $F \subseteq X$. Write $M=X \oplus Y$ for some subobject $Y$ of $M$, and denote by $u: F \rightarrow X$ the inclusion monomorphism. Then $i=\left[\begin{array}{l}u \\ 0\end{array}\right]: F \rightarrow X \oplus Y$. We claim that the following commutative square is a pullback:

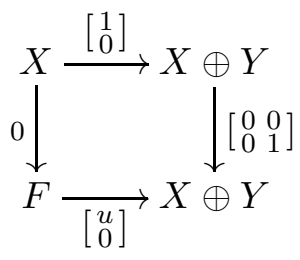

To this end, let $\alpha: Z \rightarrow F$ and $\left[\begin{array}{l}\beta_{1} \\ \beta_{2}\end{array}\right]: Z \rightarrow X \oplus Y$ be morphisms such that $\left[\begin{array}{l}u \\ 0\end{array}\right] \alpha=\left[\begin{array}{ll}0 & 0 \\ 0 & 1\end{array}\right]\left[\begin{array}{l}\beta_{1} \\ \beta_{2}\end{array}\right]$. Then $\beta_{2}=0$ and $u \alpha=0$. Hence $\alpha=0$, because $u$ is a monomorphism. It is easy to check that $\beta_{1}: Z \rightarrow X$ is the unique morphism such that $0 \beta_{1}=\alpha$ and $\left[\begin{array}{l}1 \\ 0\end{array}\right] \beta_{1}=\left[\begin{array}{l}\beta_{1} \\ \beta_{2}\end{array}\right]$. Hence the required square is a pullback.

Since $M$ is strongly self- $F$-split, it follows that the upper horizontal morphism is a fully invariant section, hence $X$ is a fully invariant direct summand of $M$. 
(ii) $\Rightarrow$ (i) Assume that (ii) holds. Consider a pullback square:

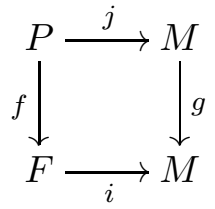

Since $M$ is self- $F$-split, $P$ is a direct summand of $M$. Since $i$ is fully invariant, there is a morphism $\alpha: F \rightarrow F$ such that $g i=i \alpha$. The pullback property yields a unique morphism $\gamma: F \rightarrow P$ such that $f \gamma=\alpha$ and $j \gamma=i$. Then $\gamma$ is a monomorphism, hence $F \subseteq P$. By hypothesis, $P$ must be a fully invariant direct summand of $M$. Hence $M$ is strongly self- $F$-split.

The following proposition generalizes [35, Proposition 2.17].

Proposition 3.6. Let $\mathcal{A}$ be an abelian category. Let $0 \rightarrow F \stackrel{i}{\rightarrow} M \stackrel{d}{\rightarrow} C \rightarrow 0$ be a fully invariant short exact sequence in $\mathcal{A}$.

(1) Assume that $M$ is (strongly) self-F-split. Let $N$ be a direct summand of $M$ with $F \subseteq N$. Then for every (fully invariant) direct summand $K$ of $M, K \cap N$ is a (fully invariant) direct summand of $M$.

(2) Assume that $M$ is dual (strongly) self-F-split. Let $N$ be a direct summand of $M$ with $N \subseteq F$. Then for every (fully invariant) direct summand $K$ of $M, K+N$ is a (fully invariant) direct summand of $M$.

Proof. (1) Denote by $u: F \rightarrow N$ the inclusion monomorphism. Write $M=N \oplus X=K \oplus Y$ for some subobjects $X, Y$ of $M$. As in the proof of Theorem [3.5, the following commutative square is a pullback:

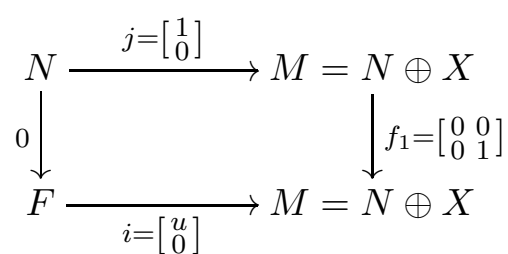

Let $k: K \cap N \rightarrow K$ and $n: K \cap N \rightarrow N$ be the inclusion monomorphisms. Consider the following commutative diagram:

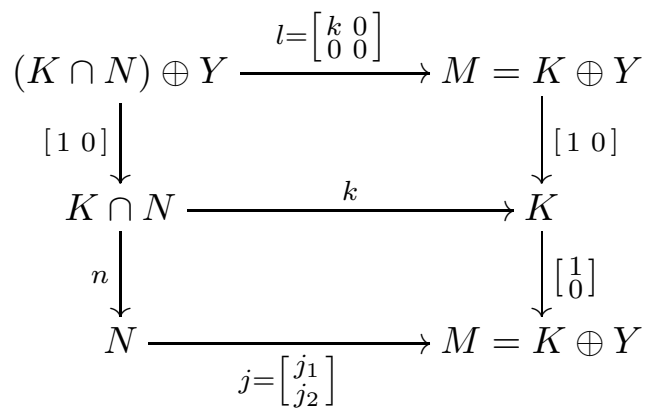

The upper and the lower squares are clearly pullbacks, hence so is the outer rectangle [20, Lemma 5.1]. Denote $f_{2}=\left[\begin{array}{ll}1 & 0 \\ 0 & 0\end{array}\right]: K \oplus Y \rightarrow K \oplus Y$. Glueing together the above 3 pullbacks, one obtains the following pullback square [20, Lemma 5.1]:

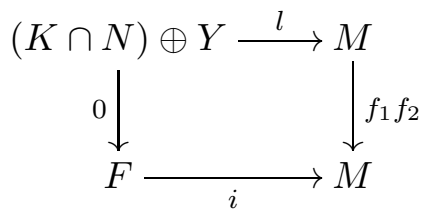

Since $M$ is (strongly) self- $F$-split, $l=\left[\begin{array}{ll}k & 0 \\ 0 & 0\end{array}\right]$ is a (fully invariant) section. It follows that $K \cap N$ is a (fully invariant) direct summand of $K$ (by Proposition 2.10), and so a (fully invariant) direct summand of $M$ (by Lemma 2.5). 
Let $\mathcal{A}$ be an abelian category. We say that an object $M$ of $\mathcal{A}$ has the (strong) summand intersection property, briefly SIP (SSIP), for a class $\mathcal{C}$ of direct summands of $M$ if the intersection of any finite family (any family) of objects from $\mathcal{C}$ belongs to $\mathcal{C}$. Dually, an object $M$ of $\mathcal{A}$ has the (strong) summand sum property, briefly $S S P(S S S P)$, for a class $\mathcal{C}$ of direct summands of $M$ if the sum of any finite family (any family) of objects from $\mathcal{C}$ belongs to $\mathcal{C}$.

Corollary 3.7. Let $\mathcal{A}$ be an abelian category. Let $0 \rightarrow F \rightarrow M \rightarrow C \rightarrow 0$ be a fully invariant short exact sequence in $\mathcal{A}$.

(1) Assume that $M$ is (strongly) self-F-split. Then:

(i) For every (fully invariant) direct summand $K$ of $M, F \cap K$ is a (fully invariant) direct summand of $M$.

(ii) $M$ has SIP for (fully invariant) direct summands containing $F$.

(2) Assume that $M$ is dual (strongly) self-F-split. Then:

(i) For every (fully invariant) direct summand $K$ of $M, F+K$ is a (fully invariant) direct summand of $M$.

(ii) $M$ has SSP for (fully invariant) direct summands contained in $F$.

Theorem 3.8. Let $\mathcal{A}$ be an abelian category. Let $0 \rightarrow F \stackrel{i}{\rightarrow} N \stackrel{d}{\rightarrow} C \rightarrow 0$ be a fully invariant short exact sequence in $\mathcal{A}$. Let $e: M \rightarrow M^{\prime}$ be an epimorphism and let $m: N^{\prime} \rightarrow N$ be a monomorphism in $\mathcal{A}$.

(1) Assume that the inclusion monomorphism $u: F \cap N^{\prime} \rightarrow N^{\prime}$ is fully invariant and $N$ is (strongly) $M-F-$-split. Then $N^{\prime}$ is (strongly) $M^{\prime}-\left(F \cap N^{\prime}\right)$-split.

(2) Assume that the induced epimorphism $q: N / N^{\prime} \rightarrow\left(\left(F+N^{\prime}\right) / N^{\prime}\right)$ is fully coinvariant and $N$ is dual (strongly) $M-F-$ split. Then $N / N^{\prime}$ is dual (strongly) $M / M^{\prime}-\left(\left(F+N^{\prime}\right) / N^{\prime}\right)$-split.

Proof. (1) Let $g: M^{\prime} \rightarrow N^{\prime}$ be a morphism in $\mathcal{A}$. Let $G=F \cap N^{\prime}$ and consider the pullback of $u$ and $g$ to get morphisms $l: Q \rightarrow M^{\prime}$ and $q: Q \rightarrow G$. Let $t: G \rightarrow F$ be the inclusion monomorphism. Consider the pullback of $i$ and mge to get morphisms $j: P \rightarrow M$ and $f: P \rightarrow$ $F$. The pullback property of the square $G N^{\prime} F N$ yields a unique morphism $h: P \rightarrow G$ such that $t h=f$ and $u h=g e j$. The pullback property of the square $Q M^{\prime} G N^{\prime}$ yields a unique morphism $p: P \rightarrow Q$ such that $q p=h$ and $l p=e j$. In this way one constructs the following commutative diagram:

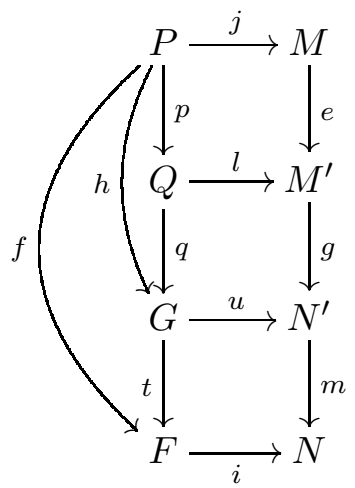

The rectangle $P M F N$ and the square $G N^{\prime} F N$ are pullbacks, hence so is the rectangle $P M G N^{\prime}$ [20. Lemma 5.1]. Since the square $Q M^{\prime} G N^{\prime}$ is a pullback, so is the square $P M Q M^{\prime}$ [20, Lemma 5.1]. Since $N$ is (strongly) $M-F$-split, $j$ is a (fully invariant) section. It is easy to check that the square $P M Q M^{\prime}$ is also a pushout, hence $l$ is a section. We may construct the following commutative diagram with exact rows:

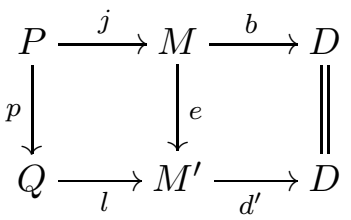


If $j$ is a fully invariant section, then $b=d^{\prime} e$ is a fully coinvariant retraction. Hence $d^{\prime}$ is a fully coinvariant retraction by Proposition 2.9, and so $l$ is a fully invariant section. Hence $N^{\prime}$ is (strongly) $M^{\prime}-\left(F \cap N^{\prime}\right)$-split.

The following corollary generalizes [35, Proposition 2.12 and Theorem 4.2].

Corollary 3.9. Let $\mathcal{A}$ be an abelian category. Let $M$ be an object and let $0 \rightarrow F \rightarrow N \rightarrow C \rightarrow 0$ be a fully invariant short exact sequence in $\mathcal{A}$. Then:

(1) $N$ is (strongly) $M$-F-split if and only if for every direct summand $M_{1}$ of $M$ and for every direct summand $N_{1}$ of $N, N_{1}$ is (strongly) $M_{1}-\left(F \cap N_{1}\right)$-split.

(2) $N$ is dual (strongly) $M-F$-split if and only if for every direct summand $M_{1}$ of $M$ and for every direct summand $N_{1}$ of $N, N / N_{1}$ is dual (strongly) $M / M_{1}-\left(\left(F+N_{1}\right) / N_{1}\right)$-split.

Proof. (1) Note that the inclusion monomorphism $u: F \cap N_{1} \rightarrow N_{1}$ is fully invariant by Corollary 2.8. Then use Theorem 3.8.

The following corollary generalizes [35, Lemma 2.10].

Corollary 3.10. Let $\mathcal{A}$ be an abelian category. Let $0 \rightarrow F \rightarrow M \rightarrow M / F \rightarrow 0$ be a fully invariant short exact sequence and $0 \rightarrow N \rightarrow M \rightarrow M / N \rightarrow 0$ a short exact sequence in $\mathcal{A}$.

(1) Assume that every morphism $N \rightarrow N$ can be extended to a morphism $M \rightarrow M$ in $\mathcal{A}$. If $M$ is (strongly) self-F-split, then $N$ is (strongly) self- $(F \cap N)$-split.

(2) Assume that every morphism $M / N \rightarrow M / N$ can be lifted to a morphism $M \rightarrow M$ in $\mathcal{A}$. If $M$ is dual (strongly) self-F-split, then $M / N$ is dual (strongly) self- $((F+N) / N)$-split.

Proof. By Proposition 2.7, the inclusion monomorphism $u: F \cap N \rightarrow N$ is fully invariant. Then use Theorem 3.8 .

Let $\mathcal{A}$ be an abelian category and let $r$ be a preradical of $\mathcal{A}$. Then one may define a preradical $r^{-1}$ of $\mathcal{A}^{\text {op }}$ by $r^{-1}(A)=A / r(A)$ for every object $A$ of $\mathcal{A}$. Recall that $r$ is called hereditary if $r$ is a left exact functor, and cohereditary if $r^{-1}$ is a right exact functor (e.g., see [2]).

Corollary 3.11. Let $\mathcal{A}$ be an abelian category and let $r$ be a preradical of $\mathcal{A}$. Let $e: M \rightarrow M^{\prime}$ be an epimorphism and let $m: N^{\prime} \rightarrow N$ be a monomorphism in $\mathcal{A}$.

(1) Assume that $r$ is hereditary and $N$ is (strongly) $M-r(N)$-split. Then $N^{\prime}$ is (strongly) $M^{\prime}-r\left(N^{\prime}\right)$-split.

(2) Assume that $r$ is cohereditary and $N$ is dual (strongly) $M-r(N)$-split. Then $N / N^{\prime}$ is dual (strongly) $M / M^{\prime}-r\left(N / N^{\prime}\right)$-split.

Proof. If $r$ is hereditary, then $r(N) \cap N^{\prime}=r\left(N^{\prime}\right)$ is fully invariant in $N^{\prime}$ [2, I.2.1]. Also, if $r$ is cohereditary, then $\left(r(N)+N^{\prime}\right) / N^{\prime}=r\left(N / N^{\prime}\right)$ is fully invariant in $N / N^{\prime}$ [2, I.2.8]. Then use Theorem 3.8.

\section{F-split objects versus Rickart objects}

Let us note that (strongly) relative $F$-split and dual (strongly) relative $F$-split objects generalize (strongly) relative Rickart and dual (strongly) relative Rickart objects defined as follows.

Definition 4.1 ([5, 7]). Let $M$ and $N$ be objects of an abelian category $\mathcal{A}$. Then $N$ is called:

(1) (strongly) $M$-Rickart if for every morphism $f: M \rightarrow N$, $\operatorname{ker}(f)$ is a (fully invariant) section.

(2) dual (strongly) $M$-Rickart if for every morphism $f: M \rightarrow N$, $\operatorname{coker}(f)$ is a (fully invariant) retraction.

(3) (strongly) self-Rickart if $N$ is (strongly) $N$-Rickart.

(4) dual (strongly) self-Rickart if $N$ is dual (strongly) $N$-Rickart.

Remark 4.2. Let $M$ and $N$ be objects of an abelian category $\mathcal{A}$. Then $N$ is (strongly) $M$ Rickart if and only if $N$ is (strongly) $M$-0-split, while $N$ is (strongly) dual $M$-Rickart if and only if $N$ is dual (strongly) $M$ - $M$-split. Also, $N$ is (strongly) self-Rickart if and only if $N$ is (strongly) self-0-split, while $N$ is (strongly) dual self-Rickart if and only if $N$ is dual (strongly) self- $N$-split. 
The following theorem generalizes [35, Theorem 2.3 and Proposition 2.4], and is one of the key results of the paper.

Theorem 4.3. Let $\mathcal{A}$ be an abelian category. Let $0 \rightarrow F \stackrel{i}{\rightarrow} M \stackrel{d}{\rightarrow} C \rightarrow 0$ be a fully invariant short exact sequence in $\mathcal{A}$.

(1) The following are equivalent:

(i) $M$ is (strongly) self-F-split.

(ii) $M \cong F \oplus C$ and $C$ is (strongly) self-Rickart.

(2) The following are equivalent:

(i) $M$ is dual (strongly) self-F-split.

(ii) $M \cong F \oplus C$ and $F$ is dual (strongly) self-Rickart.

Proof. (1) (i) $\Rightarrow$ (ii) Assume that $M$ is (strongly) self- $F$-split. Then the morphism $j: P \rightarrow M$ from the following pullback square is a (fully invariant) section:

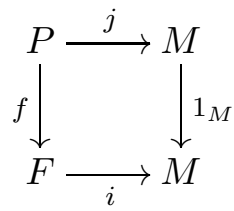

Since $f$ must be an isomorphism, it follows that $i$ is a section. Hence $M \cong F \oplus C$.

Let $g: C \rightarrow C$ be a morphism in $\mathcal{A}$ with kernel $k: K \rightarrow C$. Consider the following commutative square:

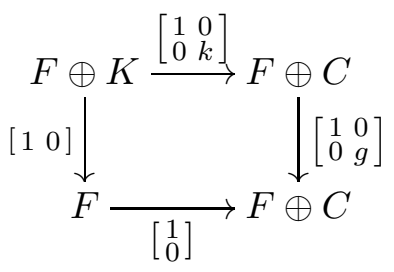

We claim that it is a pullback square. To this end, let $\alpha: Z \rightarrow F$ and $\left[\begin{array}{l}\beta_{1} \\ \beta_{2}\end{array}\right]: Z \rightarrow F \oplus C$ be morphisms in $\mathcal{A}$ such that $\left[\begin{array}{ll}1 & 0 \\ 0 & g\end{array}\right]\left[\begin{array}{l}\beta_{1} \\ \beta_{2}\end{array}\right]=\left[\begin{array}{l}1 \\ 0\end{array}\right] \alpha$, that is, $\beta_{1}=\alpha$ and $g \beta_{2}=0$. Then there exists a unique morphism $\gamma: Z \rightarrow K$ such that $\beta_{2}=k \gamma$. Consider the morphism $\left[\begin{array}{c}\beta_{1} \\ \gamma\end{array}\right]: Z \rightarrow F \oplus K$. Then $\left[\begin{array}{ll}1 & 0\end{array}\right]\left[\begin{array}{c}\beta_{1} \\ \gamma\end{array}\right]=\alpha$ and $\left[\begin{array}{ll}1 & 0 \\ 0 & k\end{array}\right]\left[\begin{array}{l}\beta_{1} \\ \gamma\end{array}\right]=\left[\begin{array}{l}\beta_{1} \\ \beta_{2}\end{array}\right]$. If $\left[\begin{array}{l}\gamma_{1} \\ \gamma_{2}\end{array}\right]: Z \rightarrow F \oplus K$ is another morphism such that $\left[\begin{array}{ll}1 & 0\end{array}\right]\left[\begin{array}{l}\gamma_{1} \\ \gamma_{2}\end{array}\right]=\alpha$ and $\left[\begin{array}{ll}1 & 0 \\ 0 & k\end{array}\right]\left[\begin{array}{l}\gamma_{1} \\ \gamma_{2}\end{array}\right]=\left[\begin{array}{l}\beta_{1} \\ \beta_{2}\end{array}\right]$, then $\gamma_{1}=\alpha=\beta_{1}$ and $k \gamma_{2}=\beta_{2}=k \gamma$. This implies that $\gamma_{2}=\gamma$, because $k$ is a monomorphism, and so $\left[\begin{array}{c}\gamma_{1} \\ \gamma_{2}\end{array}\right]=\left[\begin{array}{c}\beta_{1} \\ \gamma\end{array}\right]$. Hence the square is a pullback.

Since $M \cong F \oplus C$ is (strongly) self- $F$-split, $\left[\begin{array}{ll}1 & 0 \\ 0 & k\end{array}\right]$ is a (fully invariant) section. It follows that $k$ is a (fully invariant) section (by Proposition 2.10). Hence $C$ is (strongly) self-Rickart.

(ii) $\Rightarrow$ (i) Assume that $M \cong F \oplus C$ and $C$ is (strongly) self-Rickart. Let $f=\left[\begin{array}{ll}a & b \\ c & d\end{array}\right]: F \oplus C \rightarrow$ $F \oplus C$ be a morphism. Since $c: F \rightarrow C$ and $F$ is a fully invariant subobject of $M \cong F \oplus C$, it follows that $c=0$, hence $f=\left[\begin{array}{ll}a & b \\ 0 & d\end{array}\right]$. Denote $k=\operatorname{ker}(d): K \rightarrow C$. We have $\left[\begin{array}{ll}a & b \\ 0 & d\end{array}\right]\left[\begin{array}{ll}1 & 0 \\ 0 & k\end{array}\right]=$ $\left[\begin{array}{l}1 \\ 0\end{array}\right]\left[\begin{array}{ll}a & b k\end{array}\right]$, hence the following square is commutative:

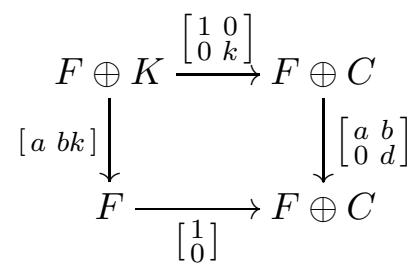

We claim that it is a pullback square. To this end, let $\alpha: Z \rightarrow F$ and $\left[\begin{array}{l}\beta_{1} \\ \beta_{2}\end{array}\right]: Z \rightarrow F \oplus C$ be morphisms in $\mathcal{A}$ such that $\left[\begin{array}{ll}a & b \\ 0 & d\end{array}\right]\left[\begin{array}{l}\beta_{1} \\ \beta_{2}\end{array}\right]=\left[\begin{array}{l}1 \\ 0\end{array}\right] \alpha$, that is, $a \beta_{1}+b \beta_{2}=\alpha$ and $d \beta_{2}=0$. Then there exists a unique morphism $\gamma: Z \rightarrow K$ such that $\beta_{2}=k \gamma$. Consider the morphism 
$\left[\begin{array}{c}\beta_{1} \\ \gamma\end{array}\right]: Z \rightarrow F \oplus K$. Then $[a b k]\left[\begin{array}{c}\beta_{1} \\ \gamma\end{array}\right]=a \beta_{1}+b k \gamma=a \beta_{1}+b \beta_{2}=\alpha$ and $\left[\begin{array}{ll}1 & 0 \\ 0 & k\end{array}\right]\left[\begin{array}{c}\beta_{1} \\ \gamma\end{array}\right]=\left[\begin{array}{c}\beta_{1} \\ k \gamma\end{array}\right]=\left[\begin{array}{c}\beta_{1} \\ \beta_{2}\end{array}\right]$. If $\left[\begin{array}{l}\gamma_{1} \\ \gamma_{2}\end{array}\right]: Z \rightarrow F \oplus K$ is another morphism such that $[a b k]\left[\begin{array}{l}\gamma_{1} \\ \gamma_{2}\end{array}\right]=\alpha$ and $\left[\begin{array}{ll}1 & 0 \\ 0 & k\end{array}\right]\left[\begin{array}{l}\gamma_{1} \\ \gamma_{2}\end{array}\right]=\left[\begin{array}{l}\beta_{1} \\ \beta_{2}\end{array}\right]$, then $\gamma_{1}=\beta_{1}$ and $k \gamma_{2}=\beta_{2}=k \gamma$. This implies that $\gamma_{2}=\gamma$, because $k$ is a monomorphism, and so $\left[\begin{array}{l}\gamma_{1} \\ \gamma_{2}\end{array}\right]=\left[\begin{array}{c}\beta_{1} \\ \gamma\end{array}\right]$. This shows that the square is a pullback.

Since $C$ is (strongly) self-Rickart, $k$ is a (fully invariant) section. It follows that $\left[\begin{array}{ll}1 & 0 \\ 0 & k\end{array}\right]$ is a section. Hence $M \cong F \oplus C$ is self- $F$-split.

If $k$ is fully invariant, then we claim that $\left[\begin{array}{ll}1 & 0 \\ 0 & k\end{array}\right]$ is also fully invariant. To this end, let $\left[\begin{array}{ll}h_{1} & h_{2} \\ h_{3} & h_{4}\end{array}\right]: F \oplus C \rightarrow F \oplus C$ be a morphism. As above, we must have $h_{3}=0$. For the morphism $h_{4}: C \rightarrow C$, there exists a morphism $\alpha: K \rightarrow K$ such that $h_{4} k=k \alpha$. Consider the morphism $\left[\begin{array}{cc}h_{1} & h_{2} k \\ 0 & \alpha\end{array}\right]: F \oplus K \rightarrow F \oplus K$. Then $\left[\begin{array}{cc}h_{1} & h_{2} \\ 0 & h_{4}\end{array}\right]\left[\begin{array}{ll}1 & 0 \\ 0 & k\end{array}\right]=\left[\begin{array}{ll}1 & 0 \\ 0 & k\end{array}\right]\left[\begin{array}{cc}h_{1} & h_{2} k \\ 0 & \alpha\end{array}\right]$, which shows that $\left[\begin{array}{ll}1 & 0 \\ 0 & k\end{array}\right]$ is fully invariant. Hence, if $C$ is strongly self-Rickart, then $M \cong F \oplus C$ is strongly self- $F$-split.

Corollary 4.4. Let $M$ be an object of an abelian category $\mathcal{A}$. Then:

(1) $M$ is (strongly) self-Rickart if and only if for every split fully invariant short exact sequence $0 \rightarrow F \rightarrow M \rightarrow C \rightarrow 0, M$ is (strongly) self-F-split.

(2) $M$ is dual (strongly) self-Rickart if and only if for every split fully invariant short exact sequence $0 \rightarrow F \rightarrow M \rightarrow C \rightarrow 0, M$ is dual (strongly) self-F-split.

Proof. (1) For the direct implication, let $0 \rightarrow F \rightarrow M \rightarrow C \rightarrow 0$ be a split fully invariant short exact sequence. Then $M \cong F \oplus C$. By [5, Corollary 2.11] ([7, Corollary 2.18]), $C$ is (strongly) self-Rickart. Hence $M$ is (strongly) self- $F$-split by Theorem 4.3 ,

Conversely, for $F=0, M$ is (strongly) self-0-split, that is, $M$ is (strongly) self-Rickart.

Example 4.5. Consider the abelian group $G=\mathbb{Z}_{p^{2}} \oplus \mathbb{Z}_{q}$, where $p$ and $q$ are distinct primes and we denote $\mathbb{Z}_{n}=\mathbb{Z} / n \mathbb{Z}$ for every natural number $n$. The subgroups of $G$ are $0, G, H_{1}$, $H_{2}, H_{3}$ and $H_{4}$, where the last 4 subgroups have orders $q, p, p q$ and $p^{2}$ respectively. Note that all subgroups of $G$ are fully invariant, while $0, G, H_{1}$ and $H_{4}$ are direct summands of $G$. Clearly, $G$ is self- $G$-split. But $G$ is not self-0-split, i.e., self-Rickart, because its direct summand $H_{4} \cong \mathbb{Z}_{p^{2}}$ is not self-Rickart [22, Theorem 5.6]. By Theorem 4.3, $G$ cannot be either self- $H_{2}$-split or self- $H_{3}$-split. Again by Theorem 4.3, $G \cong H_{1} \oplus H_{4}$ is not self- $H_{1}$-split, because $H_{4}$ is not selfRickart, while $G \cong H_{1} \oplus H_{4}$ is strongly self- $H_{4}$-split, because $H_{1} \cong \mathbb{Z}_{q}$ is strongly self-Rickart [7. Corollary 3.9]. Hence $G$ is strongly self- $F$-split for $F \in\left\{H_{4}, G\right\}$ and not self- $F$-split for $F \in\left\{0, H_{1}, H_{2}, H_{3}\right\}$. Similarly, $G$ is dual strongly self- $F$-split for $F \in\left\{H_{1}, G\right\}$ and not dual self- $F$-split for $F \in\left\{0, H_{2}, H_{3}, H_{4}\right\}$. This also shows that there are abelian groups which are self- $F$-split but not dual self- $F$-split, and viceversa.

Example 4.6. Consider the abelian group $G=\mathbb{Z}_{p} \oplus \mathbb{Z}_{p} \oplus \mathbb{Z} \oplus \mathbb{Q}$ for some prime $p$, and its subgroup $F \cong \mathbb{Z}_{p} \oplus \mathbb{Z}_{p}$. By [29, Lemma 1.9], $F$ is a fully invariant subgroup of $G$, because $\operatorname{Hom}_{\mathbb{Z}}\left(\mathbb{Z}_{p} \oplus \mathbb{Z}_{p}, \mathbb{Z} \oplus \mathbb{Q}\right)=0$. By Theorem 4.3, $G \cong F \oplus \mathbb{Z} \oplus \mathbb{Q}$ is self- $F$-split, because $\mathbb{Z} \oplus \mathbb{Q}$ is self-Rickart [24, Example 2.15]. On the other hand, $G$ is not strongly self- $F$-split, because $\mathbb{Z} \oplus \mathbb{Q}$ is not strongly self-Rickart [7, Theorem 3.6]. By Theorem 4.3, $G \cong F \oplus \mathbb{Z} \oplus \mathbb{Q}$ is dual self- $F$-split, because $F$ is clearly dual self-Rickart being semisimple. On the other hand, $G$ is not dual strongly self- $F$-split, because $F$ is not dual strongly self-Rickart [7, Theorem 3.6].

Let $\mathcal{A}$ be an abelian category and let $r$ be a preradical of $\mathcal{A}$. Recall that $r$ is called idempotent if $r r=r$, and radical if $r r^{-1}=0$. Note that every hereditary preradical is idempotent, and every cohereditary preradical is a radical (e.g., see [2]).

Example 4.7. For every abelian group $G$, denote by $t(G)$ the set of elements of $G$ having finite order (torsion elements), and by $d(G)$ the sum of its divisible (injective) subgroups. Then $t$ is a hereditary radical and $d$ is an idempotent radical of the category Ab of abelian groups. Let $G$ be a finitely generated abelian group. Then there are two classical direct sum decompositions $G=t(G) \oplus F=d(G) \oplus R$ for some subgroups $F$ (torsionfree) and $R$ (reduced) of $G$. Note that $F \cong \mathbb{Z}^{n}$ for some $n \in \mathbb{N}$, hence $F$ is projective, while $d(G)$ is injective. It follows that $G$ is self$t(G)$-split and dual self- $d(G)$-split by Theorem 4.3 and [5, Corollary 4.8]. By [7, Corollary 3.9], 
$G$ is strongly self- $t(G)$-split if and only if $F \cong \mathbb{Z}$, while $G$ is strongly dual self- $d(G)$-split if and only if $R \cong \mathbb{Z}_{p^{\infty}}$ (the Prüfer $p$-group) for some prime $p$.

Example 4.8. Consider the $\operatorname{ring} R=\left[\begin{array}{c}\mathbb{Z} Z \\ 0 \\ \mathbb{Z}\end{array}\right]$ and $M=R_{R}$. Then $F=\left[\begin{array}{l}\mathbb{Z} \mathbb{Z} \\ 0\end{array}\right]$ is an ideal of $R$, hence it is a fully invariant submodule of $M$. Moreover, we have $M=F \oplus C$, where $C=\left[\begin{array}{ll}0 & 0 \\ 0 & \mathbb{Z}\end{array}\right]$. Since $C \cong \mathbb{Z}$ is strongly self-Rickart [7, Corollary 3.9], $M$ is strongly self- $F$-split by Theorem 4.3. Note that $F$ is self-Rickart [24, Example 1.2]. If $F$ were dual self-Rickart, then it would be self-regular, and so $\operatorname{End}_{R}(F) \cong \mathbb{Z}$ would be a von Neumann regular ring, contradiction. Hence $F$ is not dual self-Rickart, and so $M$ is not dual self- $F$-split by Theorem 4.3 .

Example 4.9. Consider the ring $R=\left[\begin{array}{cc}K & K \\ 0 & K\end{array}\right]$ for some field $K$ and $M=R_{R}$. Then $F=\left[\begin{array}{cc}K & K \\ 0 & 0\end{array}\right]$ is an ideal of $R$, hence it is a fully invariant submodule of $M$. Moreover, we have $M=F \oplus C$, where $C=\left[\begin{array}{ll}0 & 0 \\ 0 & K\end{array}\right]$. Since $C \cong K$ is strongly self-Rickart and the indecomposable $F$ is strongly dual self-Rickart by [23, Example 3.9], it follows that $M$ is both strongly self- $F$-split and dual strongly self- $F$-split by Theorem 4.3 .

Now we may give another result relating strong self- $F$-splitness and self- $F$-splitness. First recall that a ring $R$ is called abelian if every idempotent element of $R$ is central.

Theorem 4.10. Let $\mathcal{A}$ be an abelian category. Let $0 \rightarrow F \rightarrow M \rightarrow C \rightarrow 0$ be a fully invariant short exact sequence in $\mathcal{A}$.

(1) $M$ is strongly self-F-split if and only if $M$ is self-F-split and $\operatorname{End}_{\mathcal{A}}(C)$ is abelian.

(2) $M$ is dual strongly self-F-split if and only if $M$ is dual self-F-split and $\operatorname{End}_{\mathcal{A}}(F)$ is abelian.

Proof. (1) Assume first that $M$ is strongly self- $F$-split. Then $M$ is self- $F$-split. Also, by Theorem 4.3. $M \cong F \oplus C$ and $C$ is strongly self-Rickart. Then $\operatorname{End}_{\mathcal{A}}(C)$ is abelian by [7, Proposition 2.14].

Conversely, assume that $M$ is self- $F$-split and $\operatorname{End}_{\mathcal{A}}(C)$ is abelian. Then $M \cong F \oplus C$ and $C$ is self-Rickart by Theorem 4.3. It follows that $C$ is strongly self-Rickart by [7, Proposition 2.14]. Hence $M$ is strongly self- $F$-split by Theorem 4.3 .

Example 4.11. Let $A=\prod_{n=1}^{\infty} \mathbb{Z}_{2}, T=\left\{\left(a_{n}\right)_{n=1}^{\infty} \in A \mid a_{n}\right.$ is eventually constant $\}$ and $I=$ $\left\{\left(a_{n}\right)_{n=1}^{\infty} \in A \mid a_{n}\right.$ is eventually zero $\}=\bigoplus_{n=1}^{\infty} \mathbb{Z}_{2}$. Consider the ring $R=\left[\begin{array}{ll}T & T / I \\ 0 & T / I\end{array}\right]$ and $M=R_{R}$. Then $F=\left[\begin{array}{cc}T & T / I \\ 0 & 0\end{array}\right]$ is an ideal of $R$, hence it is a fully invariant submodule of $M$. Moreover, we have $M=F \oplus C$, where $C=\left[\begin{array}{cc}0 & 0 \\ 0 & T / I\end{array}\right]$. Since $C$ is projective, $C$ is self-Rickart by [ $\underline{5}$, Theorem 4.7], and so $M$ is self- $F$-split by Theorem 4.3. Also, $F$ is dual self-Rickart by [23, Example 4.1], and so $M$ is dual self- $F$-split by Theorem 4.3 . Since $\operatorname{End}_{R}(C)$ and $\operatorname{End}_{R}(F)=\left[\begin{array}{cc}T & T / I \\ 0 & T / I\end{array}\right]$ are commutative, it follows that $M$ is strongly self- $F$-split and dual strongly self- $F$-split by Theorem 4.10 .

The following theorem generalizes [35, Theorem 2.9].

Theorem 4.12. Let $\mathcal{A}$ be an abelian category. Let $0 \rightarrow F \stackrel{i}{\rightarrow} M \stackrel{d}{\rightarrow} C \rightarrow 0$ be a fully invariant short exact sequence in $\mathcal{A}$.

(1) The following are equivalent:

(i) $M$ is (strongly) self-F-split and for every pullback square

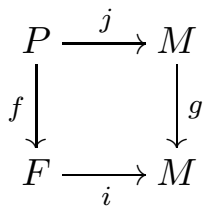

the unique morphism $l: \operatorname{Ker}(g) \rightarrow P$ is a (fully invariant) section.

(ii) $M$ is (strongly) self-Rickart and $M \cong F \oplus C$.

(2) The following are equivalent: 
(i) $M$ is dual (strongly) F-split and for every pushout square

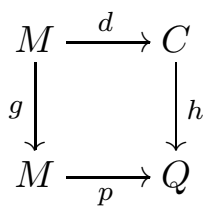

the unique morphism $q: Q \rightarrow \operatorname{Coker}(g)$ is a (fully coinvariant) retraction.

(ii) $M$ is dual (strongly) self-Rickart and $M \cong F \oplus C$.

Proof. (1) (i) $\Rightarrow$ (ii) Assume that (i) holds. Let $g: M \rightarrow M$ be a morphism in $\mathcal{A}$ with kernel $k: \operatorname{Ker}(g) \rightarrow M$. Consider the pullback of $g$ and $i$ as in the above diagram. The pullback property yields a unique morphism $l: \operatorname{Ker}(g) \rightarrow P$ such that $k=j l$. Since $M$ is (strongly) self- $F$-split, $j: P \rightarrow M$ is a (fully invariant) section. It follows that $k=j l$ is a section. If $j$ and $l$ are fully invariant sections, then so is $k=j l$ by Lemma 2.5. Hence $M$ is (strongly) self-Rickart. Also, $M \cong F \oplus C$ by Theorem 4.3 ,

(ii) $\Rightarrow$ (i) Assume that (ii) holds. Consider a pullback square as above. Denote by $k$ : $\operatorname{Ker}(g) \rightarrow M$ the kernel of $g: M \rightarrow M$. The pullback property yields a unique morphism $l: \operatorname{Ker}(g) \rightarrow P$ such that $k=j l$. Since $M \cong F \oplus C$ is (strongly) self-Rickart, so is $C$ by [5, Corollary 2.11] ([7, Corollary 2.18]). Hence $M$ is (strongly) self- $F$-split by Theorem 4.3. Since $M$ is (strongly) self-Rickart, $k=j l$ is a (fully invariant) section. It follows that $l: \operatorname{Ker}(g) \rightarrow P$ is a section. If $k=j l$ is a fully invariant section, then so is $l$ by Proposition 2.9.

The next result generalizes [35, Proposition 2.16], without the quasi-projectivity condition.

Proposition 4.13. Let $\mathcal{A}$ be an abelian category. Let $0 \rightarrow F \stackrel{i}{\rightarrow} M \stackrel{d}{\rightarrow} C \rightarrow 0$ be a fully invariant short exact sequence in $\mathcal{A}$. Then:

(1) $M$ is (strongly) self-F-split if and only if for every subobject $K$ of $M$ with $K \subseteq F, M / K$ is (strongly) self- $(F / K)$-split.

(2) $M$ is dual (strongly) self-F-split if and only if for every subobject $K$ of $M$ with $F \subseteq K$, $K$ is dual (strongly) self-F-split.

Proof. (1) Assume first that $M$ is (strongly) self- $F$-split. By Theorem 4.3, $M \cong F \oplus C$ and $C$ is (strongly) self-Rickart. Let $K$ be a subobject of $M$ with $K \subseteq F$. One may construct the following commutative diagram

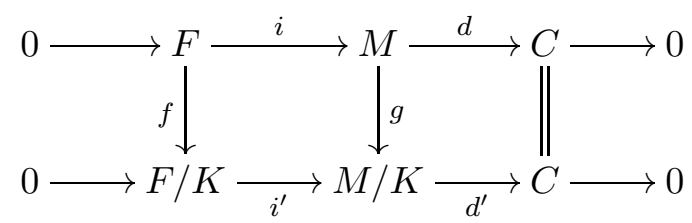

where the rows are short exact sequences and $f, g$ are the cokernels induced by the inclusions of $K$ into $F$ and $M$. Since $d=d^{\prime} g$ is a fully coinvariant retraction, so is $d^{\prime}$ by Proposition 2.9 . Then $i^{\prime}: F / K \rightarrow M / K$ is a fully invariant section, and so $M / K \cong F / K \oplus C$. Hence $M / K$ is (strongly) self- $(F / K)$-split by Theorem 4.3 .

The converse is clear.

\section{Coproducts of $F$-Split objects}

In general arbitrary coproducts of relative split objects are not relative split objects, for instance, see [35, Example 3.1]. Nevertheless, we may give the following theorem if we impose some extra conditions on the coproducts.

Theorem 5.1. Let $\mathcal{A}$ be an abelian category with coproducts, and let $\left(M_{k}\right)_{k \in K}$ be a family of objects of $\mathcal{A}$. Let $0 \rightarrow F_{k} \stackrel{i_{k}}{\rightarrow} M_{k} \stackrel{d_{k}}{\rightarrow} C_{k} \rightarrow 0$ be fully invariant short exact sequences in $\mathcal{A}$ for every $k \in K$.

(1) (i) Assume that $\operatorname{Hom}_{\mathcal{A}}\left(M_{k}, M_{l}\right)=0$ for every $k, l \in K$ with $k \neq l$. Then $\bigoplus_{k \in K} M_{k}$ is self- $\left(\bigoplus_{k \in K} F_{k}\right)$-split if and only if $M_{k}$ is self- $F_{k}$-split for every $k \in K$. 
(ii) Then $\bigoplus_{k \in K} M_{k}$ is strongly self- $\left(\bigoplus_{k \in K} F_{k}\right)$-split if and only if $M_{k}$ is strongly self$F_{k}$-split for every $k \in K$ and $\operatorname{Hom}_{\mathcal{A}}\left(C_{k}, C_{l}\right)=0$ for every $k, l \in K$ with $k \neq l$.

(2) (i) Assume that $\operatorname{Hom}_{\mathcal{A}}\left(M_{k}, M_{l}\right)=0$ for every $k, l \in K$ with $k \neq l$. Then $\bigoplus_{k \in K} M_{k}$ is dual self- $\left(\bigoplus_{k \in K} F_{k}\right)$-split if and only if $M_{k}$ is dual self- $F_{k}$-split for every $k \in K$.

(ii) Then $\bigoplus_{k \in K} M_{k}$ is dual strongly self- $\left(\bigoplus_{k \in K} F_{k}\right)$-split if and only if $M_{k}$ is dual strongly self- $F_{k}$-split for every $k \in K$ and $\operatorname{Hom}_{\mathcal{A}}\left(F_{k}, F_{l}\right)=0$ for every $k, l \in K$ with $k \neq l$.

Proof. (1) (i) The direct implication follows by Lemma 2.11 and Corollary 3.9.

Conversely, assume that $M_{k}$ is (strongly) self- $F_{k}$-split for every $k \in K$. Let $g: \bigoplus_{k \in K} M_{k} \rightarrow$ $\bigoplus_{k \in K} M_{k}$ be a morphism. Since $\operatorname{Hom}_{\mathcal{A}}\left(M_{k}, M_{l}\right)=0$, for every $k, l \in K$ with $k \neq l$, the matrix of $g$ has zero entries except for the entries $(k, k)$ with $k \in K$, which are some morphisms $g_{k}: M_{k} \rightarrow M_{k}$. Hence $g=\bigoplus_{k \in K} g_{k}$. Then we have pullback squares as follows:
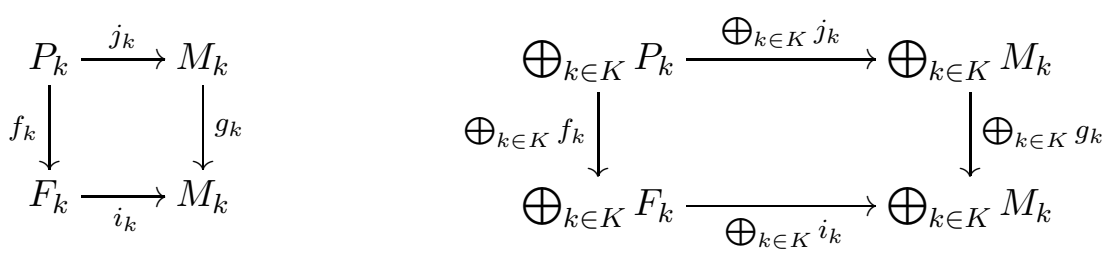

For every $k \in K, M_{k}$ is (strongly) self- $F_{k}$-split, hence $j_{k}$ is a (fully invariant) section. It follows that $\bigoplus_{k \in K} j_{k}$ is a (fully invariant) section (by Lemma 2.11). Hence $\bigoplus_{k \in K} M_{k}$ is (strongly) self- $\left(\bigoplus_{k \in K} F_{k}\right)$-split.

(ii) By [7, Theorem 3.6], $\bigoplus_{k \in K} C_{k}$ is strongly self-Rickart if and only if $C_{k}$ is strongly selfRickart and $\operatorname{Hom}_{\mathcal{A}}\left(C_{k}, C_{l}\right)=0$ for every $k, l \in K$ with $k \neq l$. Then use (i) and Theorem 4.3 to derive the conclusion.

Example 5.2. Let us see that the zero Hom conditions from Theorem 5.1 are not superfluous.

(a) Consider the abelian group $G=G_{1} \oplus G_{2}$, where $G_{1}=\mathbb{Z}_{q} \oplus \mathbb{Z}$ and $G_{2}=\mathbb{Z}_{p}$ for some distinct primes $p$ and $q$. By [29, Lemma 1.9], $F_{1} \cong \mathbb{Z}_{q}$ is a fully invariant subgroup of $G_{1}$, because $\operatorname{Hom}_{\mathbb{Z}}\left(\mathbb{Z}_{q}, \mathbb{Z}\right)=0$. Clearly, $F_{2}=0$ is a fully invariant subgroup of $G_{2}$. By Theorem 4.3, $G_{1} \cong F_{1} \oplus \mathbb{Z}$ is strongly self- $F_{1}$-split and $G_{2}=\mathbb{Z}_{p}$ is strongly self- $F_{2}$-split, because $\mathbb{Z}$ and $\mathbb{Z}_{p}$ are strongly self-Rickart [7, Corollary 3.9]. Note that $F_{1} \oplus F_{2}=\mathbb{Z}_{q}$ is a fully invariant subgroup of $G=G_{1} \oplus G_{2}$, because $\operatorname{Hom}_{\mathbb{Z}}\left(\mathbb{Z}_{q}, \mathbb{Z} \oplus \mathbb{Z}_{p}\right)=0$. By Theorem 4.3, $G$ is not self- $F_{1} \oplus F_{2}$-split, because $\mathbb{Z} \oplus \mathbb{Z}_{p}$ is not self-Rickart [22, Example 2.5]. Note that $\operatorname{Hom}_{\mathbb{Z}}\left(G_{1}, G_{2}\right) \neq 0$.

(b) Consider the abelian group $G=G_{1} \oplus G_{2}$, where $G_{1}=\mathbb{Z}_{p^{\infty}} \oplus \mathbb{Z}_{q}$ and $G_{2}=\mathbb{Z}_{p}$ for some distinct primes $p$ and $q$. By [29, Lemma 1.9], $F_{1} \cong \mathbb{Z}_{p^{\infty}}$ is a fully invariant subgroup of $G_{1}$, because $\operatorname{Hom}_{\mathbb{Z}}\left(\mathbb{Z}_{p^{\infty}}, \mathbb{Z}_{q}\right)=0$. Clearly, $F_{2}=\mathbb{Z}_{p}$ is a fully invariant subgroup of $G_{2}$. By Theorem 4.3. $G_{1} \cong F_{1} \oplus \mathbb{Z}_{q}$ is dual strongly self- $F_{1}$-split and $G_{2}=\mathbb{Z}_{p}$ is dual strongly 0 -split, because $F_{1} \cong \mathbb{Z}_{p^{\infty}}$ and $\mathbb{Z}_{p}$ are dual strongly self-Rickart [7, Corollary 3.9]. Note that $F_{1} \oplus F_{2}=\mathbb{Z}_{p^{\infty}} \oplus \mathbb{Z}_{p}$ is a fully invariant subgroup of $G=G_{1} \oplus G_{2}$, because $\operatorname{Hom}_{\mathbb{Z}}\left(\mathbb{Z}_{p} \infty \oplus \mathbb{Z}_{p}, \mathbb{Z}_{q}\right)=0$. By Theorem 4.3. $G$ is not dual self- $F_{1} \oplus F_{2}$-split, because $\mathbb{Z}_{p^{\infty}} \oplus \mathbb{Z}_{p}$ is not self-Rickart [23, Example 2.10]. Note that $\operatorname{Hom}_{\mathbb{Z}}\left(G_{2}, G_{1}\right) \neq 0$.

Theorem 5.3. Let $\mathcal{A}$ be an abelian category. Let $M$ and $N$ be objects of $\mathcal{A}, N=\bigoplus_{k=1}^{n} N_{k}$ a direct sum decomposition, and $0 \rightarrow F \rightarrow N \rightarrow C \rightarrow 0$ a fully invariant short exact sequence in A. Then:

(1) $N$ is (strongly) $M-F$-split if and only if $N_{k}$ is (strongly) $M-\left(F \cap N_{k}\right)$-split for every $k \in\{1, \ldots, n\}$.

(2) $N$ is dual (strongly) $M-F$-split if and only if $N_{k}$ is dual (strongly) $M-\left(\left(F+N_{k}\right) / N_{k}\right)$-split for every $k \in\{1, \ldots, n\}$.

Proof. (1) The direct implication follows by Corollary 3.9.

For the sake of clarity, we prove the converse for $n=2$, the general case following inductively. Assume that $N_{k}$ is (strongly) $M$ - $\left(F \cap N_{k}\right)$-split for $k=1,2$. By Proposition [2.6 we have $F \cong\left(F \cap N_{1}\right) \oplus\left(F \cap N_{2}\right)$. Denote by $i=\left[\begin{array}{cc}i_{1} & 0 \\ 0 & i_{2}\end{array}\right]:\left(F \cap N_{1}\right) \oplus\left(F \cap N_{2}\right) \rightarrow N_{1} \oplus N_{2}$ the inclusion 
morphism. Let $g=\left[\begin{array}{l}g_{1} \\ g_{2}\end{array}\right]: M \rightarrow N=N_{1} \oplus N_{2}$ be a morphism in $\mathcal{A}$. Consider the following pullback squares:
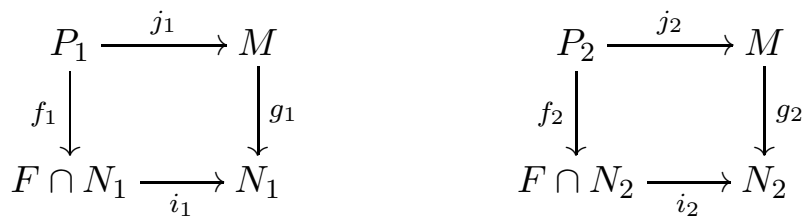

Since $N_{1}$ is (strongly) $M$ - $\left(F \cap N_{1}\right)$-split and $N_{2}$ is (strongly) $M$ - $\left(F \cap N_{2}\right)$-split, $j_{1}$ and $j_{2}$ are (fully invariant) sections. Then there exists an epimorphism $p_{2}: M \rightarrow P_{2}$ such that $p_{2} j_{2}=1_{P_{2}}$. Consider the pullback of $g_{1} j_{2}: P_{2} \rightarrow N_{1}$ and $i_{1}$ in order to get the outer part of the following commutative diagram:

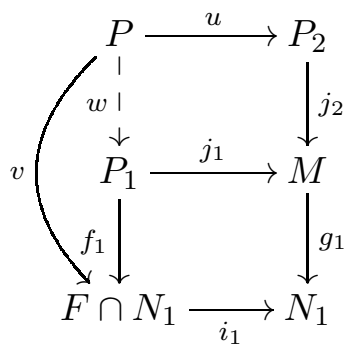

By Theorem 3.8, $N_{1}$ is $P_{2}-F_{1}$-split, hence $u$ is a (fully invariant) section. Since the lower square is a pullback, so is the upper square by [20, Lemma 5.1]. Also, there exists a unique morphism $w: P \rightarrow P_{1}$ such that $f_{1} w=v$ and $j_{1} w=j_{2} u$.

We claim that the following square is a pullback:

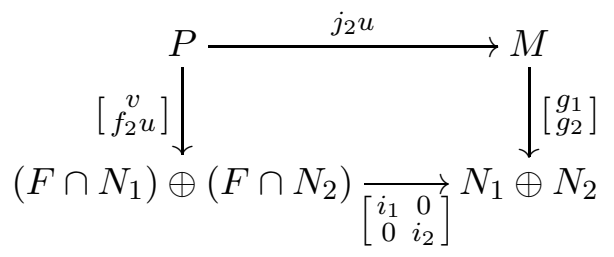

It is commutative, because we have

$$
\left[\begin{array}{cc}
i_{1} & 0 \\
0 & i_{2}
\end{array}\right]\left[\begin{array}{c}
v \\
f_{2} u
\end{array}\right]=\left[\begin{array}{c}
i_{1} v \\
i_{2} f_{2} u
\end{array}\right]=\left[\begin{array}{l}
g_{1} j_{2} u \\
g_{2} j_{2} u
\end{array}\right]=\left[\begin{array}{l}
g_{1} \\
g_{2}
\end{array}\right] j_{2} u .
$$

Now let $\left[\begin{array}{c}\alpha_{1} \\ \alpha_{2}\end{array}\right]: X \rightarrow\left(F \cap N_{1}\right) \oplus\left(F \cap N_{2}\right)$ and $\beta: X \rightarrow M$ be morphisms such that $\left[\begin{array}{cc}i_{1} & 0 \\ 0 & i_{2}\end{array}\right]\left[\begin{array}{l}\alpha_{1} \\ \alpha_{2}\end{array}\right]=$ $\left[\begin{array}{l}g_{1} \\ g_{2}\end{array}\right] \beta$. Hence $i_{1} \alpha_{1}=g_{1} \beta$ and $i_{2} \alpha_{2}=g_{2} \beta$. The pullback properties of the first two squares from the proof yield unique morphisms $\gamma_{1}: X \rightarrow P_{1}$ such that $f_{1} \gamma_{1}=\alpha_{1}$ and $j_{1} \gamma_{1}=\beta$, and $\gamma_{2}: X \rightarrow P_{2}$ such that $f_{2} \gamma_{2}=\alpha_{2}$ and $j_{2} \gamma_{2}=\beta$. Hence $j_{1} \gamma_{1}=j_{2} \gamma_{2}$. Since the square $P P_{2} P_{1} M$ is a pullback, there exists a unique morphism $\gamma: X \rightarrow P$ such that $w \gamma=\gamma_{1}$ and $u \gamma=\gamma_{2}$. It follows that $\left[\begin{array}{c}v \\ f_{2} u\end{array}\right] \gamma=\left[\begin{array}{l}f_{1} w \gamma \\ f_{2} \gamma_{2}\end{array}\right]=\left[\begin{array}{l}f_{1} \gamma_{1} \\ f_{2} \gamma_{2}\end{array}\right]=\left[\begin{array}{l}\alpha_{1} \\ \alpha_{2}\end{array}\right]$ and $j_{2} u \gamma=j_{2} \gamma_{2}=\beta$. For uniqueness, if there exists a morphism $\gamma^{\prime}: X \rightarrow P$ such that $\left[\begin{array}{c}v \\ f_{2} u\end{array}\right] \gamma^{\prime}=\left[\begin{array}{c}\alpha_{1} \\ \alpha_{2}\end{array}\right]$ and $j_{2} u \gamma^{\prime}=\beta$, then we have $j_{2} u \gamma=j_{2} u \gamma^{\prime}$. Then $\gamma=\gamma^{\prime}$, because $j_{2}$ and $u$ are monomorphisms. Thus, the required square is a pullback.

Finally, since $j_{2}$ and $u$ are (fully invariant) sections, so is $j_{2} u$ (by Lemma 2.5). This shows that $N$ is (strongly) $M$ - $F$-split.

Corollary 5.4. Let $\mathcal{A}$ be an abelian category. Let $M$ and $N$ be objects of $\mathcal{A}, N=\bigoplus_{k \in K} N_{k}$ a direct sum decomposition, and $0 \rightarrow F \rightarrow N \rightarrow C \rightarrow 0$ a fully invariant short exact sequence in $\mathcal{A}$. Then:

(1) Assume that $M$ is finitely generated. Then $N$ is (strongly) $M-F$-split if and only if $N_{k}$ is (strongly) $M-\left(F \cap N_{k}\right)$-split for every $k \in K$.

(2) Assume that $N$ is finitely cogenerated. Then $N$ is dual (strongly) $M-F-$-split if and only if $N_{k}$ is dual (strongly) $M-\left(\left(F+N_{k}\right) / N_{k}\right)$-split for every $k \in K$.

Proof. (1) The direct implication follows by Corollary 3.9. 
Conversely, assume that $N_{k}$ is (strongly) $M$ - $\left(F \cap N_{k}\right)$-split for every $k \in K$. Let $g: M \rightarrow$ $N=\bigoplus_{k \in K} N_{k}$ be a morphism in $\mathcal{A}$. Since $M$ is finitely generated, we may write $g=l g^{\prime}$ for some morphism $g^{\prime}: M \rightarrow \bigoplus_{k \in F} N_{k}$ and inclusion morphism $l: \bigoplus_{k \in A} N_{k} \rightarrow N$, where $A$ is a finite subset of $K$. Consider the following commutative diagram:

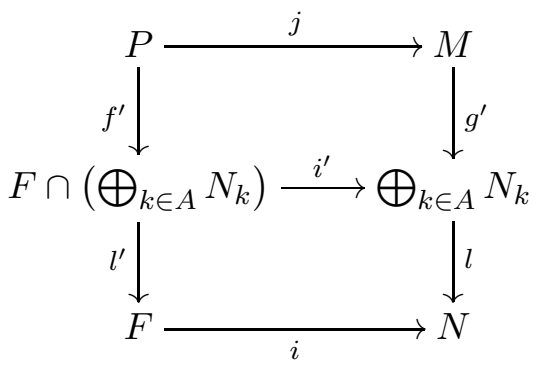

in which the lower and the upper rectangles are pullbacks. Then the outer rectangle is a pullback [20, Lemma 5.1]. By Corollary [2.8, $i^{\prime}$ is a fully invariant kernel. By Theorem [5.3, $\bigoplus_{k \in A} N_{k}$ is (strongly) $M-\left(F \cap\left(\bigoplus_{k \in A} N_{k}\right)\right)$-split, hence $j$ is a (fully invariant) section. It follows that $N$ is (strongly) $M-F$-split.

Remark 5.5. Note that in general Theorem 5.3 does not hold for arbitrary coproducts. For an example in the case $F=0$ see [5, Example 3.3].

Let $\mathcal{A}$ be an abelian category and let $r$ be a preradical of $\mathcal{A}$. Recall that an object $A$ of $\mathcal{A}$ is called $r$-torsion if $r(A)=A$, and $r$-torsionfree if $r(A)=0$. The class of $r$-torsion objects is closed under factor objects and coproducts, while the class of $r$-torsionfree objects is closed under subobjects and products. The preradical $r$ is called superhereditary if $r$ is hereditary and the class of $r$-torsion objects of $\mathcal{A}$ is closed under products (e.g., see [2]).

Theorem 5.6. Let $\mathcal{A}$ be an abelian category and let $r$ be a preradical of $\mathcal{A}$.

(1) Let $M$ be an object of $\mathcal{A}$ and let $\left(N_{k}\right)_{k \in K}$ be a family of objects of $\mathcal{A}$ having a coproduct.

(i) Assume that $M$ has SSIP for (fully invariant) direct summands containing $r(M)$. Then $\bigoplus_{k \in K} N_{k}$ is (strongly) $M-r\left(\bigoplus_{k \in K} N_{k}\right)$-split if and only if $N_{k}$ is (strongly) $M-r\left(N_{k}\right)$-split for every $k \in K$.

(ii) Assume that $K$ is finite and $M$ has SIP for (fully invariant) direct summands containing $r(M)$. Then $\bigoplus_{k \in K} N_{k}$ is (strongly) $M-r\left(\bigoplus_{k \in K} N_{k}\right)$-split if and only if $N_{k}$ is (strongly) $M-r\left(N_{k}\right)$-split for every $k \in K$.

(2) Let $N$ be an object of $\mathcal{A}$ and let $\left(M_{k}\right)_{k \in K}$ be a family of objects of $\mathcal{A}$ having a product.

(i) Assume that $r$ is superhereditary and $N$ has SSSP for (fully invariant) direct summands contained in $r(N)$. Then $N$ is dual (strongly) $\prod_{k \in K} M_{k}-r(N)$-split if and only if $N$ is dual (strongly) $M_{k}-r(N)$-split for every $k \in K$.

(ii) Assume that $K$ is finite and $N$ has SSP for (fully invariant) direct summands contained in $r(N)$. Then $N$ is dual (strongly) $\prod_{k \in K} M_{k}-r(N)$-split if and only if $N$ is dual (strongly) $M_{k}-r(N)$-split for every $k \in K$.

Proof. (1) (i) The direct implication follows by Corollary 3.9.

Conversely, assume that $N_{k}$ is (strongly) $M-r\left(N_{k}\right)$-split for every $k \in K$. Denote by $i$ : $r\left(\bigoplus_{k \in K} N_{k}\right) \rightarrow \bigoplus_{k \in K} N_{k}$ the inclusion monomorphism and by $d: \bigoplus_{k \in K} N_{k} \rightarrow C$ its cokernel. Let $g: M \rightarrow \bigoplus_{k \in K} N_{k}$ be a morphism in $\mathcal{A}$. Consider the pullback of $g$ and $i$ in order to get morphisms $f: P \rightarrow r\left(\bigoplus_{k \in K} N_{k}\right)$ and $j: P \rightarrow M$. For every $k \in K$, denote by $p_{k}: \bigoplus_{k \in K} N_{k} \rightarrow N_{k}$ the canonical projection and $g_{k}=p_{k} g: M \rightarrow N_{k}$. For every $k \in K$, denote by $i_{k}: r\left(N_{k}\right) \rightarrow N_{k}$ the inclusion monomorphism and by $d_{k}: N_{k} \rightarrow C_{k}$ its cokernel. For every $k \in K$, consider the following pullback diagram:

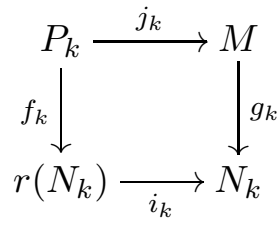


Let $u: r(M) \rightarrow M$ be the inclusion monomorphism. Since $r$ is a preradical, each morphism $g_{k}$ : $M \rightarrow N_{k}$ induces a morphism $l_{k}: r(M) \rightarrow r\left(N_{k}\right)$ such that $g_{k} u=i_{k} l_{k}$. By the pullback property of the upper square it follows that $r(M) \subseteq P_{k}$ for every $k \in K$. Since each $N_{k}$ is (strongly) $M-r\left(N_{k}\right)$-split, each $j_{k}: P_{k} \rightarrow M$ is a (fully invariant) section. Note that $r\left(\bigoplus_{k \in K} N_{k}\right)=$ $\bigoplus_{k \in K} r\left(N_{k}\right)$ [2, I.1.2]. It follows that

$$
\operatorname{Ker}(d g)=P=\bigcap_{k \in K} P_{k}=\bigcap_{k \in K} \operatorname{Ker}\left(d_{k} g_{k}\right)
$$

and $M$ has SSIP for (fully invariant) direct summands containing $r(M)$, it follows that $j=$ $\operatorname{ker}(d g): P \rightarrow M$ is a (fully invariant) section. Hence $\bigoplus_{k \in K} N_{k}$ is (strongly) $M-r\left(\bigoplus_{k \in K} N_{k}\right)$ split by Lemma 3.2 .

(ii) This is similar to (i).

(2) Let us only note that if $r$ is superhereditary, then $r\left(\prod_{k \in K} M_{k}\right)=\prod_{k \in K} r\left(M_{k}\right)$ by definition and [2, I.1.2]. Then the proof is dual to (1).

Corollary 5.7. Let $\mathcal{A}$ be an abelian category, let $r$ be a preradical of $\mathcal{A}$, let $M_{1}, \ldots, M_{k}$ be objects of $\mathcal{A}$ and $l \in\{1, \ldots, n\}$.

(1) $\bigoplus_{k=1}^{n} M_{k}$ is (strongly) $M_{l}-r\left(\bigoplus_{k=1}^{n} M_{k}\right)$-split if and only if $M_{k}$ is (strongly) $M_{l}-r\left(M_{k}\right)$ split for every $k \in\{1, \ldots, n\}$.

(2) $M_{l}$ is dual (strongly) $\bigoplus_{k=1}^{n} M_{k}-r\left(M_{l}\right)$-split if and only if $M_{l}$ is dual (strongly) $M_{k}-r\left(M_{l}\right)$ split for every $k \in\{1, \ldots, n\}$.

Proof. This follows by Corollary 3.7 and Theorem 5.6 .

\section{Classes all of whose objects are $F$-split}

Recall that an abelian category $\mathcal{A}$ is called spectral if every short exact sequence in $\mathcal{A}$ splits.

Theorem 6.1. Let $\mathcal{A}$ be an abelian category.

(1) The following are equivalent:

(i) $\mathcal{A}$ is spectral.

(ii) $\mathcal{A}$ has enough injectives and $N$ is $M-F$-split for every objects $M$ and $N$ of $\mathcal{A}$ and for every fully invariant subobject $F$ of $N$.

(iii) $\mathcal{A}$ has enough injectives and every object $N$ of $\mathcal{A}$ is self-F-split for every fully invariant subobject $F$ of $N$.

(iv) $\mathcal{A}$ has enough injectives and $N$ is $M-F$-split for every objects $M$ and $N$ of $\mathcal{A}$ with $N$ injective and for every fully invariant subobject $F$ of $N$.

(v) $\mathcal{A}$ has enough injectives and every injective object $N$ of $\mathcal{A}$ is self-F-split for every fully invariant subobject $F$ of $N$.

(2) The following are equivalent:

(i) $\mathcal{A}$ is spectral.

(ii) $\mathcal{A}$ has enough projectives and $N$ is dual $M-F$-split for every objects $M$ and $N$ of $\mathcal{A}$ and for every fully invariant subobject $F$ of $N$.

(iii) $\mathcal{A}$ has enough projectives and every object $N$ of $\mathcal{A}$ is dual self-F-split for every fully invariant subobject $F$ of $N$.

(iv) $\mathcal{A}$ has enough projectives and $N$ is dual $M$-Rickart for every objects $M$ and $N$ of $\mathcal{A}$ with $M$ projective and for every fully invariant subobject $F$ of $N$.

(v) $\mathcal{A}$ has enough projectives and every projective object $N$ of $\mathcal{A}$ is dual self-F-split for every fully invariant subobject $F$ of $N$.

Proof. (1) (i) $\Rightarrow$ (ii) $\Rightarrow($ iii) $\Rightarrow$ (v) and (ii) $\Rightarrow$ (iv) $\Rightarrow$ (v) are clear.

(v) $\Rightarrow(\mathrm{i})$ Assume that $\mathcal{A}$ has enough injectives and every injective object $N$ of $\mathcal{A}$ is self- $F$-split for every fully invariant subobject $F$ of $N$. Taking $F=0$, it follows that every injective object $N$ of $\mathcal{A}$ is self-Rickart, hence $\mathcal{A}$ is spectral by [5, Theorem 4.1].

The category $\operatorname{Mod}(R)$ is a locally finitely generated (i.e., it has a family of finitely generated generators) Grothendieck category with enough injectives and enough projectives. It is spectral if and only if $R$ is semisimple [32, Chapter V, Proposition 6.7]. 
Corollary 6.2. Let $R$ be a unitary ring. Then the following are equivalent:

(i) $R$ is semisimple.

(ii) Every right $R$-module $N$ is (strongly) self-F-split for every fully invariant submodule $F$ of $N$.

(iii) Every injective right $R$-module $N$ is (strongly) self-F-split for every fully invariant submodule $F$ of $N$.

(iv) Every right $R$-module $N$ is dual (strongly) self-F-split for every fully invariant submodule $F$ of $N$.

(v) Every projective right $R$-module $N$ is dual (strongly) self-F-split for every fully invariant submodule $F$ of $N$.

Proof. We only discuss the strong versions of the equivalences (i) $\Leftrightarrow($ ii $) \Leftrightarrow($ iii), the remaining part following by Theorem 6.1 and by duality.

(i) $\Rightarrow$ (ii) Assume that $R$ is semisimple. Then every right $R$-module is semisimple, hence for every right $R$-module $N$, every submodule of $N$ is fully invariant. Thus every right $R$-module is (weak) duo [29]. By Theorem 6.1, every right $R$-module is self-0-split, i.e., self-Rickart. Then every right $R$-module is strongly self-Rickart [7, Corollary 2.10]. Now using Theorem [4.3, it follows that every right $R$-module $N$ is strongly self- $F$-split for every fully invariant submodule $F$ of $N$.

(ii) $\Rightarrow$ (iii) This is clear.

(iii) $\Rightarrow$ (i) This follows by Theorem 6.1.

Let ${ }^{C} \mathcal{M}$ be the category of left comodules over a coalgebra $C$ over a field [10]. Then ${ }^{C} \mathcal{M}$ is a locally finite (i.e., it has a family of generators of finite length) Grothendieck category with enough injectives. It has enough projectives if and only if $C$ is left semiperfect [10, Theorem 3.2.3]. It is spectral if and only if ${ }^{C} \mathcal{M}$ is semisimple if and only if $C$ is cosemisimple. Now one can deduce the following corollary similarly to Corollary 6.2 .

Corollary 6.3. Let $C$ be a coalgebra over a field. Then the following are equivalent:

(i) $C$ is cosemisimple.

(ii) Every left $C$-comodule $N$ is (strongly) self-F-split for every fully invariant subcomodule $F$ of $N$.

(iii) Every injective left $C$-comodule $N$ is (strongly) self-F-split for every fully invariant subcomodule $F$ of $N$.

(iv) $C$ is left semiperfect and every left $C$-comodule $N$ is dual (strongly) self-F-split for every fully invariant subcomodule $F$ of $N$.

(v) $C$ is left semiperfect and every projective left $C$-comodule $N$ is dual (strongly) self-F-split for every fully invariant subcomodule $F$ of $N$.

A Grothendieck category $\mathcal{A}$ is called a $V$-category if every simple object of $\mathcal{A}$ is injective [13], and a regular category if every object $B$ of $\mathcal{A}$ is regular in the sense that every short exact sequence of the form $0 \rightarrow A \rightarrow B \rightarrow C \rightarrow 0$ is pure in $\mathcal{A}$ (i.e., every finitely presented object is projective with respect to it) [40, p. 313].

Theorem 6.4. Let $\mathcal{A}$ be a locally finitely generated Grothendieck category. Then:

(1) $\mathcal{A}$ is a $V$-category if and only if every finitely cogenerated (injective) object $N$ of $\mathcal{A}$ is self-F-split for every fully invariant subobject $F$ of $N$.

(2) $\mathcal{A}$ is a regular category if and only if every finitely generated (projective) object $N$ of $\mathcal{A}$ is dual self-F-split for every finitely generated fully invariant subobject $F$ of $N$.

Proof. (1) If $\mathcal{A}$ is a $V$-category, then $\mathcal{A}$ has a semisimple cogenerator [13, Theorem 2.3]. Hence every finitely cogenerated (injective) object $N$ of $\mathcal{A}$ is semisimple, and so $N$ is self- $F$-split for every fully invariant subobject $F$ of $N$.

The remaining part of the proof follows by [5, Theorem 4.4], using Remark 4.2 ,

(2) We give its proof, since it is not completely dual to (1).

Assume that $\mathcal{A}$ is a regular category. Let $N$ be a finitely generated object of $\mathcal{A}, F$ a finitely generated fully invariant subobject of $N$ and $g: N \rightarrow N$ a morphism in $\mathcal{A}$. Then $\operatorname{Im}(g i)$ is a 
finitely generated subobject of the regular object $N$, hence $\operatorname{Im}(g i)$ is a direct summand of $N$ by [40, 37.4], whose proof works in locally finitely generated Grothendieck categories. Hence $\operatorname{coker}(g i)$ is a retraction, and so $N$ is dual self- $F$-split by Lemma 3.2 .

The remaining part of the proof follows by [ $\underline{5}$, Theorem 4.4], using Remark 4.2.

Corollary 6.5. Let $R$ be a unitary ring. Then:

(1) $R$ is a right $V$-ring if and only if every finitely cogenerated (injective) right $R$-module $N$ is self-F-split for every fully invariant submodule $F$ of $N$.

(2) $R$ is a von Neumann regular ring if and only if every finitely generated (projective) right $R$-module $N$ is dual self-F-split for every finitely generated fully invariant submodule $F$ of $N$.

Proof. Note that $\operatorname{Mod}(R)$ is a $V$-category if and only if $R$ is a right $V$-ring, and a regular category if and only if $R$ is a von Neumann regular ring. Then use Theorem 6.4.

Remark 6.6. If $R$ is a commutative unitary ring, then $V$-rings coincide with von Neumann regular rings [40, 23.5], hence all statements from Corollary 6.5 are equivalent.

Corollary 6.7. Let $C$ be a coalgebra over a field. Then the following are equivalent:

(i) $C$ is cosemisimple.

(ii) Every finitely cogenerated (injective) left $C$-comodule $N$ is self-F-split for every fully invariant subcomodule $F$ of $N$.

(iii) Every finitely generated (projective) left $C$-comodule is dual self-F-split for every finitely generated fully invariant subcomodule $F$ of $N$.

Proof. Note that the comodule category ${ }^{C} \mathcal{M}$ is a $V$-category if and only if ${ }^{C} \mathcal{M}$ is a regular category if and only if $C$ is cosemisimple by [38, Proposition 2.3] and the proof of [5, Corollary 4.6]. Then use Theorem 6.4.

Recall that an abelian category $\mathcal{A}$ is called (semi)hereditary if every (finitely generated) subobject of a projective object is projective, and co(semi)hereditary if every (finitely cogenerated) factor object of an injective object is injective.

Theorem 6.8. Let $\mathcal{A}$ be an abelian category.

(1) Assume that $\mathcal{A}$ has enough projectives. Then $\mathcal{A}$ is (semi)hereditary if and only if every (finitely generated) projective object $N$ of $\mathcal{A}$ is self-F-split for every fully invariant subobject $F$ of $N$.

(2) Assume that $\mathcal{A}$ has enough injectives. Then $\mathcal{A}$ is co(semi)hereditary if and only if every (finitely cogenerated) injective object $N$ of $\mathcal{A}$ is dual self-F-split for every fully invariant subobject $F$ of $N$.

Proof. (1) Assume that $\mathcal{A}$ is (semi)hereditary. Let $N$ be a (finitely generated) projective object of $\mathcal{A}$, and $0 \rightarrow F \stackrel{i}{\rightarrow} N \stackrel{d}{\rightarrow} C \rightarrow 0$ a fully invariant short exact sequence in $\mathcal{A}$. Let $g: N \rightarrow N$ be a morphism in $\mathcal{A}$. If $N$ is finitely generated, then so is $\operatorname{Im}(d g)$. By Theorem 4.3 we have $N \cong F \oplus C$, hence $C$ is (finitely generated) projective. Since $\mathcal{A}$ is (semi)hereditary, $\operatorname{Im}(d g) \subseteq C$ is projective. Then $\operatorname{Ker}(d g)$ is a direct summand of $N$, and so $N$ is self- $F$-split by Lemma 3.2 ,

The remaining part of the proof follows by [5, Theorem 4.7], using Remark 4.2.

Corollary 6.9. Let $R$ be a unitary ring. Then the following are equivalent:

(i) $R$ is right hereditary.

(ii) Every projective right $R$-module $N$ is self-F-split for every fully invariant submodule $F$ of $N$.

(iii) Every injective right $R$-module $N$ is dual self-F-split for every fully invariant submodule $F$ of $N$.

Proof. Note that $\operatorname{Mod}(R)$ is (co)hereditary if and only if the ring $R$ is right hereditary. Then use Theorem 6.8.

Corollary 6.10. Let $C$ be a coalgebra over a field. Then: 
(1) If $C$ is left semiperfect, then $C$ is hereditary if and only if every projective left $C$-comodule $N$ is self-F-split for every fully invariant subcomodule $F$ of $N$.

(2) $C$ is hereditary if and only if every injective left $C$-comodule $N$ is dual self-F-split for every fully invariant subcomodule $F$ of $N$.

Proof. Note that the comodule category ${ }^{C} \mathcal{M}$ is (co)hereditary if and only if $C$ is a (left and right) hereditary coalgebra [28. Then use Theorem 6.8 .

Next we give some results in the case of abelian categories with a (projective) generator or an (injective) cogenerator. Recall that an object $M$ of an abelian category is called (semi)hereditary if every (finitely generated) subobject of $M$ is projective, and co(semi)hereditary if every (finitely cogenerated) factor object of $M$ is injective.

Theorem 6.11. Let $\mathcal{A}$ be an abelian category, and let $r$ be a preradical of $\mathcal{A}$.

(1) Assume that $\mathcal{A}$ has a generator $G$ and enough injectives, and $r$ is a radical. Then the following are equivalent:

(i) $G / r(G)$ is a (semi)hereditary object of $\mathcal{A}$.

(ii) $G=r(G) \oplus B$ for some (semi)hereditary object $B$ of $\mathcal{A}$.

(iii) For every (finite) direct sum $M=G^{(I)}, M=r(M) \oplus D$ for some (semi) hereditary object $D$ of $\mathcal{A}$.

(iv) For every (finitely generated) projective object $M, M=r(M) \oplus D$ for some (semi)hereditary object $D$ of $\mathcal{A}$.

(v) $r(G)$ is a direct summand of $G$ and every (finitely generated) $r$-torsionfree projective object of $\mathcal{A}$ is (semi)hereditary.

(vi) $r(G)$ is a direct summand of $G$ and every (finitely generated) $r$-torsionfree projective object of $\mathcal{A}$ is self-Rickart.

(vii) Every (finite) direct sum $M=G^{(I)}$ is self-r(M)-split.

(viii) Every (finitely generated) projective object $M$ is self-r $(M)$-split.

(2) Assume that $\mathcal{A}$ has a cogenerator $G$ and enough projectives. Then the following are equivalent:

(i) $r(G)$ is a cosemihereditary object of $\mathcal{A}$.

(ii) $r(G)$ is a cosemihereditary direct summand of $G$.

(iii) For every finite direct sum $M=G^{n}, r(M)$ is a cosemihereditary direct summand of $M$.

(iv) For every finitely cogenerated injective object $M, r(M)$ is a cosemihereditary direct summand of $M$.

(v) $r(G)$ is a direct summand of $G$ and every finitely cogenerated $r$-torsion injective object of $\mathcal{A}$ is cosemihereditary.

(vi) $r(G)$ is a direct summand of $G$ and every finitely cogenerated $r$-torsion injective object of $\mathcal{A}$ is dual self-Rickart.

(vii) Every finite direct sum $M=G^{n}$ is dual self-r $(M)$-split.

(viii) Every finitely cogenerated injective object $M$ is dual self-r(M)-split.

Proof. (1) (i) $\Rightarrow$ (ii) Assume that $G / r(G)$ is a (semi)hereditary object of $\mathcal{A}$. It follows that $G / r(G)$ is projective, and so $G=r(G) \oplus B$ with $B \cong G / r(G)$ (semi)hereditary.

(ii) $\Rightarrow$ (iii) Assume that $G=r(G) \oplus B$ for some (semi)hereditary object $B$ of $\mathcal{A}$. Let $M=G^{(I)}$ for some (finite) set $I$. Then $M=r(G)^{(I)} \oplus B^{(I)}=r(M) \oplus B^{(I)}$. Since $B$ is (semi)hereditary, so is $B^{(I)}$ by [40, 39.3, 39.7], whose proofs work in abelian categories with enough injectives.

(iii) $\Rightarrow$ (iv) Assume that (iii) holds. Let $M$ be a (finitely generated) projective object of $\mathcal{A}$. Then $M$ is a direct summand of a (finite) direct sum $F=G^{(I)}$, say $F=M \oplus N$. It follows that $r(F)=r(M) \oplus r(N)$ and $F / r(F) \cong M / r(M) \oplus N / r(N)$. Since $F / r(F)$ is (semi)hereditary, so is $M / r(M)$. Since $r(F)$ is a direct summand of $F, r(M)$ must be a direct summand of $M$.

(iv) $\Rightarrow$ (v) This is clear.

(v) $\Rightarrow$ (vi) Assume that (v) holds. Let $M$ be a (finitely generated) $r$-torsionfree projective object of $\mathcal{A}$. Let $f: M \rightarrow M$ be a morphism in $\mathcal{A}$. Since $M$ is (semi)hereditary, $M / \operatorname{Ker}(f) \cong$ $\operatorname{Im}(f)$ is projective, and so $\operatorname{Ker}(f)$ is a direct summand of $M$. Hence $M$ is self-Rickart. 
(vi) $\Rightarrow$ (vii) Assume that (vi) holds. Let $M=G^{(I)}$ be a (finite) direct sum. Since $G=r(G) \oplus B$, it follows that $M=r(M) \oplus B^{(I)}$. Since the (finitely generated) $r$-torsionfree projective object $B^{(I)}$ is self-Rickart, $M$ is self- $r(M)$-split by Theorem 4.3 .

(vii) $\Rightarrow$ (viii) This follows by Corollary 3.9 .

(viii) $\Rightarrow$ (i) Assume that (viii) holds. By Theorem 4.3, $G=r(G) \oplus B$ for some self-Rickart object $B$ of $\mathcal{A}$. We show that $B$ is (semi)hereditary. To this end, let $A$ be a (finitely generated) subobject of $B$. Then $A \cong M / K$ for some (finite) direct sum $M=G^{(I)}$ and subobject $K$ of $M$. Denote by $\varphi: A \rightarrow M / K$ an isomorphism, by $p: M \rightarrow M / K$ the natural epimorphism, by $k: A \rightarrow M$ and $i: r(M) \rightarrow M$ the inclusion monomorphisms and $g=k \varphi p: M \rightarrow M$. Consider the pullback of $i$ and $g$ in order to get the following commutative square:

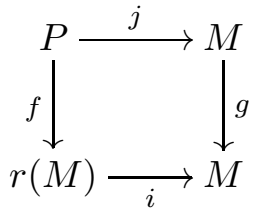

Since $r$ is a radical, $B \cong G / r(G)$ is an $r$-torsionfree object, hence so is $A$. This implies that if $=g j=0$. Then $p j=0$, and so there exists a unique morphism $\alpha: P \rightarrow K$ such that $k \alpha=j$, which implies that $\alpha$ is a monomorphism. The pullback property yields a unique morphism $\beta: K \rightarrow P$ such that $f \beta=0$ and $j \beta=k$. Then $k \alpha \beta=k$, hence $\alpha \beta=1_{K}$, which shows that $P \cong K$. Since $M$ is projective, it is self- $r(M)$-split. Then $j: P \rightarrow M$ is a section, and so $A$ is projective. Hence $G / r(G) \cong B$ is (semi)hereditary.

(2) In general only the semihereditary case from (1) may be dualized, because $r$ may not preserve products (unless it is superhereditary), and an arbitrary product of cohereditary objects may not be cohereditary (see [40, 18.2] and the proofs of [40, 39.3, 39.6, 39.7]).

Corollary 6.12. Let $r$ be a radical of $\operatorname{Mod}(R)$. Then the following are equivalent:

(i) $R / r(R)$ is a (semi)hereditary right $R$-module.

(ii) $R=r(R) \oplus B$ for some (semi)hereditary right ideal $B$ of $R$.

(iii) For every (finitely generated) free right $R$-module $M, M=r(M) \oplus D$ for some (semi) hereditary right $R$-module $D$.

(iv) For every (finitely generated) projective right $R$-module $M, M=r(M) \oplus D$ for some (semi)hereditary right $R$-module $D$.

(v) $r(R)$ is a direct summand of $R$ and every (finitely generated) $r$-torsionfree projective right $R$-module is (semi)hereditary.

(vi) $r(R)$ is a direct summand of $R$ and every (finitely generated) $r$-torsionfree projective right $R$-module is self-Rickart.

(vii) Every (finitely generated) free right $R$-module $M$ is self-r $(M)$-split.

(viii) Every (finitely generated) projective right $R$-module $M$ is self-r $(M)$-split.

Proof. This follows by Theorem 6.11, noting that $R$ is a generator of $\operatorname{Mod}(R)$.

Corollary 6.13. Let $C$ be left semiperfect and let $r$ be a preradical of ${ }^{C} \mathcal{M}$. Then the following are equivalent:

(i) $r(C)$ is a cosemihereditary left $C$-comodule.

(ii) $r(C)$ is a cosemihereditary direct summand of $C$.

(iii) For every finitely cogenerated free left $C$-comodule $M, r(M)$ is a cosemihereditary direct summand of $M$.

(iv) For every finitely cogenerated injective left $C$-comodule $M, r(M)$ is a cosemihereditary direct summand of $M$.

(v) $r(C)$ is a direct summand of $C$ and every finitely cogenerated $r$-torsion injective left $C$ comodule is cosemihereditary.

(vi) $r(C)$ is a direct summand of $C$ and every finitely cogenerated $r$-torsion injective left $C$ comodule is dual self-Rickart.

(vii) Every finitely cogenerated free left $C$-comodule $M$ is dual self-r(M)-split.

(viii) Every finitely cogenerated injective left $C$-comodule $M$ is dual self- $r(M)$-split. 
Proof. This follows by Theorem 6.11, noting that $C$ is a cogenerator of ${ }^{C} \mathcal{M}$, and ${ }^{C} \mathcal{M}$ has enough projectives if and only if $C$ is left semiperfect.

Theorem 6.14. Let $\mathcal{A}$ be an abelian category and let $r$ be a preradical of $\mathcal{A}$.

(1) Assume that $\mathcal{A}$ has an injective cogenerator $G$. Then the following are equivalent:

(i) $G$ is a (strongly) self-r $(G)$-split object of $\mathcal{A}$.

(ii) $G=r(G) \oplus D$ for some object $D$ of $\mathcal{A}$ and $\operatorname{End}_{\mathcal{A}}(D)$ is a von Neumann regular (strongly regular) ring.

(iii) Every finite direct product $M=G^{n}$ is (strongly) self-r $(M)$-split.

(iv) Every finitely cogenerated injective object $M$ of $\mathcal{A}$ is (strongly) self- $r(M)$-split.

(2) Assume that $\mathcal{A}$ has a projective generator $G$. Then the following are equivalent:

(i) $G$ is a dual (strongly) self-r $(G)$-split object.

(ii) $r(G)$ is a direct summand of $G$ and $\operatorname{End}_{\mathcal{A}}(r(G))$ is a von Neumann regular (strongly regular) ring.

(iii) Every finite direct sum $M=G^{n}$ is dual (strongly) self-r $(M)$-split.

(iv) Every finitely generated projective object $M$ of $\mathcal{A}$ is dual (strongly) self-r $(M)$-split.

Proof. (1) We first prove the theorem without strongness conditions.

(i) $\Rightarrow$ (ii) Assume that (i) holds. Then $G=r(G) \oplus D$ for some self-Rickart object $D$ of $\mathcal{A}$ by Theorem 4.3. We claim that $D$ is also dual self-Rickart. To this end, let $f: D \rightarrow D$ be a morphism in $\mathcal{A}$. Then $\operatorname{Im}(f) \cong D / \operatorname{Ker}(f)$ is isomorphic to a direct summand of $D$. As $G$ is injective, so is $D$. Hence $D$ is direct injective, in the sense that every subobject of $D$ isomorphic to a direct summand of $D$ is a direct summand of $D$. It follows that $\operatorname{Im}(f)$ is a direct summand of $D$. Hence $D$ is self-Rickart and dual self-Rickart. Then $\operatorname{End}_{\mathcal{A}}(D)$ is von Neumann regular by [5. Corollary 2.3].

(ii) $\Rightarrow$ (iii) Assume that (ii) holds. Let $M=G^{n}$ for some $n \in \mathbb{N}$. Then $M=r(G)^{n} \oplus D^{n}=$ $r(M) \oplus D^{n}$. Note that $\operatorname{End}_{\mathcal{A}}\left(D^{n}\right) \cong \operatorname{End}_{\mathcal{A}}(D)^{n}$ is von Neumann regular, hence $D^{n}$ is self-regular, and so $D^{n}$ is self-Rickart by [5, Corollary 2.3]. Finally, $M$ is self- $r(M)$-split by Theorem 4.3.

(iii) $\Rightarrow$ (iv) Assume that (iii) holds. Note that every finitely cogenerated injective object of $\mathcal{A}$ is isomorphic to a direct summand of a direct product $G^{n}$. Then use Corollary 3.9.

(iv) $\Rightarrow$ (i) This is clear.

Finally, the equivalence of the strong versions of the above conditions is immediately deduced by the above proof and the following properties: an object $M$ of $\mathcal{A}$ is strongly self- $r(M)$-split if and only if $M$ is self- $r(M)$-split and $\operatorname{End}_{\mathcal{A}}(r(M))$ is abelian (Theorem 4.10), while a ring is strongly regular if and only if it is von Neumann regular and abelian.

Corollary 6.15. Let $r$ be a preradical of ${ }^{C} \mathcal{M}$. Then the following are equivalent:

(i) $C$ is a (strongly) self-r $(C)$-split left $C$-comodule.

(ii) $C=r(C) \oplus D$ for some subcomodule $D$ of $C$ and $\operatorname{End}_{C}(D)$ is a von Neumann regular (strongly regular) ring.

(iii) Every finitely cogenerated free left $C$-comodule $M$ is (strongly) self-r( $M)$-split.

(iv) Every finitely cogenerated injective left $C$-comodule $M$ is (strongly) self-r $(M)$-split.

Proof. This follows by Theorem 6.14, noting that $C$ is an injective cogenerator of ${ }^{C} \mathcal{M}$.

Corollary 6.16. Let $r$ be a preradical of $\operatorname{Mod}(R)$. Then the following are equivalent:

(i) $R$ is a dual (strongly) self-r $(R)$-split right $R$-module.

(ii) $r(R)$ is a direct summand of $R$ and $\operatorname{End}_{R}(r(R))$ is a von Neumann regular (strongly regular) ring.

(iii) Every finitely generated free right $R$-module $M$ is dual (strongly) self-r $(M)$-split.

(iv) Every finitely generated projective right $R$-module $M$ is dual (strongly) self-r( $M)$-split.

Proof. This follows by Theorem 6.14, noting that $R$ is a projective generator of $\operatorname{Mod}(R)$.

\section{FURTHER APPLiCATIONS}

Throughout the paper we have given several examples and consequences of our results. In this section we present some further applications to module and comodule categories. 
7.1. Module categories. Throughout $R$ is a unitary ring.

Proposition 7.1. $\quad$ (1) Let $I$ be a right $T$-nilpotent ideal of $R$ and let $r$ be the cohereditary radical associated to $I$, given by $r(M)=M I$. Then a right $R$-module $M$ is (strongly) self-r $(M)$-split if and only if $M$ is $r$-torsionfree (strongly) self-Rickart.

(2) Let $I$ be a left $T$-nilpotent ideal of $R$ and let $r$ be the superhereditary preradical associated to $I$, given by $r(M)=\{x \in M \mid x I=0\}$. Then a right $R$-module $M$ is dual (strongly) self-r $(M)$-split if and only if $M$ is $r$-torsion (strongly) dual self-Rickart.

Proof. (1) There is a bijective correspondence between the ideals $I$ of a ring $R$ and the cohereditary radicals $r$ of $\operatorname{Mod}(R)$, where $r$ is given by $r(M)=M I$ [2, Corollary I.2.11]. If $M$ is (strongly) self- $r(M)$-split, then $r(M)$ is a superfluous direct summand of $M$ by [2, Proposition I.4.6] and Theorem 4.3, hence $r(M)=0$, and so $M$ is $r$-torsionfree (strongly) self-Rickart. The converse is clear.

(2) There is a bijective correspondence between the ideals $I$ of a ring $R$ and the superhereditary preradicals $r$ of $\operatorname{Mod}(R)$, where $r$ is given by $r(M)=\{x \in M \mid x I=0\}$ [2, I.2.E6]. If $M$ is dual (strongly) self- $r(M)$-split, then $r(M)$ is an essential direct summand of $M$ by [2, Proposition I.4.12] and Theorem [4.3, hence $r(M)=M$, and so $M$ is $r$-torsion (strongly) dual self-Rickart. The converse is clear.

For a right $R$-module $M$, denote by $\operatorname{Soc}(M)$ the socle of $M$ (i.e., the sum of its simple submodules), and by $\operatorname{Rad}(M)$ the Jacobson radical of $M$ (i.e., the intersection of its maximal submodules). Then Soc is a hereditary preradical and Rad is a cohereditary preradical of $\operatorname{Mod}(R)$.

Proposition 7.2. $\quad$ (1) A finitely generated right $R$-module $M$ is (strongly) self-Rad( $M)$-split if and only if $M$ is semiprimitive (strongly) self-Rickart.

(2) A finitely cogenerated right $R$-module $M$ is dual (strongly) self-Soc( $M)$-split if and only if $M$ is semisimple.

Proof. (1) If $M$ is finitely generated (strongly) self- $\operatorname{Rad}(M)$-split, then $\operatorname{Rad}(M)$ is a superfluous direct summand of $M$ by Theorem 4.3, hence $\operatorname{Rad}(M)=0$, and so $M$ is semiprimitive (strongly) self-Rickart. The converse is clear.

(2) If $M$ is finitely cogenerated dual (strongly) self-Soc $(M)$-split, then $\operatorname{Soc}(M)$ is an essential direct summand of $M$ by Theorem 4.3 , hence $M=\operatorname{Soc}(M)$ is semisimple. The converse is clear.

Corollary 7.3. $\quad$ (1) Every finitely generated right $R$-module $M$ is (strongly) self- $\operatorname{Rad}(M)$ split if and only if every finitely generated right $R$-module $M$ is semiprimitive (strongly) self-Rickart.

(2) Every finitely cogenerated right $R$-module $M$ is dual (strongly) self-Soc(M)-split if and only if $R$ is a right $V$-ring.

Proof. (2) Note that every finitely cogenerated right $R$-module is semisimple if and only if $R$ is a right $V$-ring [40, 23.1].

Recall that an object $M$ of an abelian category $\mathcal{A}$ is called (strongly) extending if every subobject of $M$ is essential in a (fully invariant) direct summand of $M$, and (strongly) lifting if every subobject $L$ of $M$ contains a (fully invariant) direct summand $K$ of $M$ such that $L / K$ is superfluous in $M / K$ [5, 15, 39].

For a right $R$-module $M$, consider the full subcategory $\sigma[M]$ of $\operatorname{Mod}(R)$ consisting of $M$ subgenerated right $R$-modules. Note that if $M=R_{R}$, then $\sigma[M]=\operatorname{Mod}(R)$. For every module $N \in \sigma[M]$, denote by:

- $Z_{M}(N)=\operatorname{Tr}(\mathcal{U}, N)=\sum\{\operatorname{Im}(f) \mid f \in \operatorname{Hom}(\mathcal{U}, N)\}$, where $\mathcal{U}$ is the class of $M$-singular modules. Recall that a module $A$ is called $M$-singular (or singular in $\sigma[M]$ ) if $A \cong L / K$ for some $L \in \sigma[M]$ and essential submodule $K$ of $L$. Also, $A$ is called non-M-singular (or non-singular in $\sigma[M]$ ) if $Z_{M}(N)=0$.

- $Z_{M}^{2}(N)$ the second $M$-singular submodule of $N$, which is determined by the equality $Z_{M}^{2}(N) / Z_{M}(N)=Z_{M}\left(N / Z_{M}(N)\right)$. 
- $\bar{Z}_{M}(N)=\operatorname{Re}(N, \mathcal{U})=\bigcap\{\operatorname{Ker}(f) \mid f \in \operatorname{Hom}(N, \mathcal{U})\}$, where $\mathcal{U}$ is the class of $M$-small modules. Recall that a module $A$ is called $M$-small if $A$ is superfluous in some $L \in \sigma[M]$. Also, $N$ is called $M$-cosingular (or cosingular in $\sigma[M]$ ) if $\bar{Z}_{M}(N)=0$, and non-Mcosingular (or non-cosingular in $\sigma[M]$ ) if $\bar{Z}_{M}(N)=N$.

- $\bar{Z}_{M}^{2}(N)=\bar{Z}_{M}\left(\bar{Z}_{M}(N)\right)$.

Then $Z_{M}$ is a hereditary preradical, $Z_{M}^{2}$ is a hereditary radical, while $\bar{Z}_{M}$ and $\bar{Z}_{M}^{2}$ are radicals of $\sigma[M]$.

Proposition 7.4. (i) Assume that $R$ is non-singular (i.e., $Z\left(R_{R}\right)=0$ ) and every finitely generated non-singular right $R$-module is projective. Then every finitely generated right $R$-module $M$ is self- $Z^{2}(M)$-split.

(ii) Assume that every finitely cogenerated singular right $R$-module is injective. Then every finitely cogenerated right $R$-module $M$ is dual self- $Z(M)$-split.

Proof. (i) Let $M$ be a finitely generated right $R$-module. Since the finitely generated nonsingular (see [37, Lemma 3.2]) right $R$-module $M / Z^{2}(M)$ is projective, $M \cong Z^{2}(M) \oplus M / Z^{2}(M)$. Since every ring with the property from hypothesis is right semihereditary [17, Theorem 5.18], $M / Z^{2}(M)$ is self-Rickart [5, Theorem 4.7]. Hence $M$ is self- $Z^{2}(M)$-split by Theorem 4.3 .

(ii) Let $M$ be a finitely cogenerated right $R$-module. Since the finitely cogenerated singular right $R$-module $Z(M)$ is injective, it is a direct summand of $M$. We claim that $R$ is right cosemihereditary. To this end, let $E$ be an injective right $R$-module and $K$ a submodule of $E$ such that $E / K$ is finitely cogenerated. Consider the injective hull $E(K)$ of $K$ and the induced epimorphism $\gamma: E / K \rightarrow E / E(K)$. Then $\operatorname{Ker}(\gamma) \cong E(K) / K$ is finitely cogenerated singular, and so it is injective. Also, we have $E \cong E(K) \oplus E / E(K)$, and so $E / E(K)$ is injective. It follows that $E / K$ is injective, which shows that $R$ is right cosemihereditary. Then $Z(M)$ is dual self-Rickart [5, Theorem 4.7]. Hence $M$ is dual self- $Z(M)$-split by Theorem 4.3 ,

Proposition 7.5. Let $M$ be a right $R$-module. Then:

(1) Every (strongly) extending module $N \in \sigma[M]$ is (strongly) self- $Z_{M}^{2}(N)$-split.

(2) Every (strongly) lifting module $N \in \sigma[M]$ is dual (strongly) self- $\bar{Z}_{M}^{2}(N)$-split.

Proof. (1) If $N$ is (strongly) extending, then $N=Z_{M}^{2}(N) \oplus N^{\prime}$ for some non- $M$-singular (strongly) extending submodule $N^{\prime}$ of $N$ [37, Theorem 3.4] ([15, Theorem 2.24]). Note that a module $X \in \sigma[M]$ is non- $M$-singular if and only if for every $K \in \sigma[M]$ and for every $0 \neq f \in \operatorname{Hom}\left(K, N^{\prime}\right), \operatorname{Ker}(f)$ is not essential in $K$. Clearly, every non- $M$-singular module $X \in \sigma[M]$ is $X$ - $\mathcal{K}$-nonsingular, in the sense that for every $0 \neq f \in \operatorname{End}(X), \operatorname{Ker}(f)$ is not essential in $X$ [5, Definition 9.4]. It follows that $N^{\prime}$ is (strongly) self-Baer by [5, Theorem 9.5], and so $N^{\prime}$ is (strongly) self-Rickart. Hence $N$ is (strongly) self- $Z_{M}^{2}(N)$-split by Theorem 4.3,

(2) If $N$ is (strongly) lifting, then $N=\bar{Z}_{M}^{2}(N) \oplus N^{\prime}$ for some submodule $N^{\prime}$ of $N$ such that $\bar{Z}_{M}^{2}(N)$ is non- $M$-cosingular (strongly) lifting [33, Theorem 4.1] ([39, Corollary 3.6]). Note that a module $Y \in \sigma[M]$ is non- $M$-cosingular if and only if every non-zero factor module of $Y$ is not $M$-small, that is, it does not exist any module $L \in \sigma[M]$ such that $Y$ is superfluous in $L$. Clearly, every non- $M$-cosingular module $Y \in \sigma[M]$ is $Y$ - $\mathcal{T}$-nonsingular, in the sense that for every $0 \neq f \in \operatorname{End}(Y), \operatorname{Im}(f)$ is not superfluous in $Y$ [5, Definition 9.4]. It follows that $\bar{Z}_{M}^{2}(N)$ is dual (strongly) self-Baer by [5, Theorem 9.5], and so $\bar{Z}_{M}^{2}(N)$ is dual (strongly) self-Rickart. Hence $N$ is dual (strongly) self- $\bar{Z}_{M}^{2}(N)$-split by Theorem 4.3 ,

7.2. Comodule categories. Throughout $C$ is a coalgebra over a field $k$. Let $C^{*}=\operatorname{Hom}_{k}(C, k)$. Then $\sigma\left[C_{C^{*}}\right]=\operatorname{Rat}\left(\operatorname{Mod}\left(C^{*}\right)\right) \cong{ }^{C} \mathcal{M}$, where $\operatorname{Rat}\left(\operatorname{Mod}\left(C^{*}\right)\right)$ is the full subcategory of $\operatorname{Mod}\left(C^{*}\right)$ consisting of rational right $C^{*}$-modules and ${ }^{C} \mathcal{M}$ is the category of left $C$-comodules [10, Chapter 2]. Hence one has the (pre)radicals from categories of the form $\sigma[M]$ for some module $M$ available in comodule categories. Also, for every right $C^{*}$-module $M$, denote by $\operatorname{Rat}(M)$ the largest rational submodule of $M$. Then Rat is a hereditary preradical of $\operatorname{Mod}\left(C^{*}\right)$.

Proposition 7.6. Let $M$ be a left $C$-comodule. Then the following are equivalent:

(i) $M$ is semisimple. 
(ii) $M$ is (strongly) self-Soc( $M)$-split.

(iii) $M$ is dual (strongly) self-Soc(M)-split.

Proof. If $M$ is non-zero (strongly) self-Soc $(M)$-split or dual (strongly) self-Soc( $M)$-split, then $\operatorname{Soc}(M)$ is a direct summand of $M$ by Theorem 4.3 and an essential subcomodule of $M$ by [10. Corollary 2.4.12], which implies that $M=\operatorname{Soc}(M)$ is semisimple by [10, Proposition 2.4.11]. The rest of the proof is clear.

Corollary 7.7. The following are equivalent:

(i) $C$ is cosemisimple.

(ii) Every left $C$-comodule $M$ is (strongly) self-Soc( $M)$-split.

(iii) Every left $C$-comodule $M$ is dual (strongly) self-Soc( $M)$-split.

Proposition 7.8. Let $C$ be such that $C^{*}$ is semisimple. Then the following are equivalent:

(i) $C$ is cosemisimple.

(ii) Every right $C^{*}$-module $M$ is (strongly) self-Rat $(M)$-split.

(iii) Every right $C^{*}$-module $M$ is dual (strongly) self-Rat( $\left.M\right)$-split.

Proof. If every right $C^{*}$-module $M$ is (strongly) self-Rat $(M)$-split or dual (strongly) self-Rat $(M)$ split, then $\operatorname{Rat}(M)$ is a direct summand of $M$ for every every right $C^{*}$-module $M$ by Theorem 4.3. Then $C$ is finite dimensional by [27, Theorem 3.4]. Since $C^{*}$ is semisimple, it follows that $C$ is cosemisimple [10, Exercise 3.1.7]. The rest of the proof is clear.

Proposition 7.9. Let $C$ be hereditary and let $P$ be a projective left $C$-comodule. Then $P^{*}=$ $\operatorname{Hom}_{k}(P, k)$ is a dual self-Rat $\left(C^{*} P^{*}\right)$-split right $C$-comodule.

Proof. By [10, Corollary 2.4.18], $\operatorname{Rat}\left(C^{*} P^{*}\right)$ is an injective right $C$-comodule. Then $\operatorname{Rat}\left(C^{*} P^{*}\right)$ is a direct summand of $P^{*}$ and dual self-Rickart by [5, Corollary 4.9]. Hence $P^{*}$ is a dual self-Rat $\left(C^{*} P^{*}\right)$-split right $C$-comodule by Theorem 4.3 ,

Proposition 7.10. Let $C$ be left semiperfect hereditary and let $F$ be a flat left $C$-comodule. Then $F^{*}=\operatorname{Hom}_{k}(F, k)$ is a dual self-Rat $\left(C^{*} F^{*}\right)$-split right $C$-comodule.

Proof. By [9, Theorem 4.6] and [8, Corollary 3.4], $C$ is left semiperfect if and only if Rat $\left(C^{*} F^{*}\right)$ is an injective right $C$-comodule for every every flat left $C$-comodule $F$. Then $\operatorname{Rat}\left(C^{*} F^{*}\right)$ is a direct summand of $F^{*}$ and dual self-Rickart by [5, Corollary 4.9]. Hence $F^{*}$ is a dual self$\operatorname{Rat}\left(C^{*} F^{*}\right)$-split right $C$-comodule by Theorem 4.3 ,

\section{REFERENCES}

[1] Sh. Asgari and A. Haghany, t-Rickart and dual t-Rickart modules, Algebra Colloq. 22 (Spec 1) (2015), 849-870.

[2] L. Bican, T. Kepka and P. Nemec, Rings, modules and preradicals, Marcel Dekker, New York, 1982.

[3] S.U. Chase, Direct products of modules, Trans. Amer. Math. Soc. 97 (1960), 457-473.

[4] S. Crivei, D. Keskin Tütüncü and R. Tribak, Transfer of splitness with respect to a fully invariant short exact sequence in abelian categories, preprint, 2018.

[5] S. Crivei and A. Kör, Rickart and dual Rickart objects in abelian categories, Appl. Categ. Structures 24 (2016), 797-824.

[6] S. Crivei and G. Olteanu, Rickart and dual Rickart objects in abelian categories: Transfer via functors, Appl. Categ. Structures, 2018. DOI: 10.1007/s10485-017-9509-8

[7] S. Crivei and G. Olteanu, Strongly Rickart objects in abelian categories, Comm. Algebra, 2018. DOI: 10.1080/00927872.2018.1439046.

[8] S. Crivei, M. Prest and B. Torrecillas, Covers in finitely accessible categories, Proc. Amer. Math. Soc. 138 (2010), 1213-1221.

[9] J. Cuadra and D. Simson, Flat comodules and perfect coalgebras, Comm. Algebra 35 (2007), 3164-3194.

[10] S. Dăscălescu, C. Năstăsescu and Ş. Raianu, Hopf algebras. An introduction, Marcel Dekker, New York, 2001.

[11] S. Dăscălescu, C. Năstăsescu and A. Tudorache, A note on regular objects in Grothendieck categories, Arab. J. Sci. Eng. 36 (2011), 957-962.

[12] S. Dăscălescu, C. Năstăsescu, A. Tudorache and L. Dăuş, Relative regular objects in categories, Appl. Categ. Structures 14 (2006), 567-577.

[13] L. Dăuş, C. Năstăsescu and F. Van Oystaeyen, V-categories: applications to graded rings, Comm. Algebra 37 (2009), 3248-3258. 
[14] S. Ebrahimi Atani, M. Khoramdel and S. Dolati Pish Hesari, T-Rickart modules, Colloq. Math. 128 (2012), $87-100$.

[15] S. Ebrahimi Atani, M. Khoramdel and S. Dolati Pish Hesari, On strongly extending modules, Kyungpook Math. J. 54 (2014), 237-247.

[16] S. Ebrahimi Atani, M. Khoramdel and S. Dolati Pish Hesari, T-dual Rickart modules, Bull. Iranian Math. Soc. 42 (2016), 627-642.

[17] K. R. Goodearl, Ring theory: nonsingular rings and modules, Marcel Dekker, New York, 1976.

[18] A. Hattori, A foundation of torsion theory for modules over general rings, Nagoya Math. J. 17 (1960), $147-158$.

[19] I. Kaplansky, Modules over Dedekind rings and valuation rings, Trans. Amer. Math. Soc. 72 (1952), 327-340.

[20] G. M. Kelly, Monomorphisms, epimorphisms and pull-backs, J. Austral. Math. Soc. 9 (1969), 124-142.

[21] D. Keskin Tütüncü and R. Tribak, On dual Baer modules, Glasgow Math. J. 52 (2010), 261-269.

[22] G. Lee, S. T. Rizvi and C. Roman, Rickart modules, Comm. Algebra 38 (2010), 4005-4027.

[23] G. Lee, S. T. Rizvi and C. Roman, Dual Rickart modules, Comm. Algebra 39 (2011), 4036-4058.

[24] G. Lee, S. T. Rizvi and C. Roman, Direct sums of Rickart modules, J. Algebra 353 (2012), 62-78.

[25] S. Maeda, On a ring whose principal right ideals generated by idempotents form a lattice, J. Sci. Hiroshima Univ. Ser. A 24 (1960), 509-525.

[26] B. Mitchell, Theory of categories, Academic Press, New York, 1965.

[27] C. Năstăsescu and B. Torrecillas, The splitting problem for coalgebras, J. Algebra 281 (2004), 144-149.

[28] C. Năstăsescu, B. Torrecillas and Y. H. Zhang, Hereditary coalgebras, Comm. Algebra 24 (1996), 1521-1528.

[29] A. Ç. Özcan, A. Harmancı and P. F. Smith, Duo modules, Glasgow Math. J. 48 (2006), 533-545.

[30] C. E. Rickart, Banach algebras with an adjoint operation, Ann. of Math. 47 (1946), 528-550.

[31] S. T. Rizvi and C. Roman, Baer and quasi-Baer modules, Comm. Algebra 32 (2004), 103-123.

[32] B. Stenström, Rings of quotients, Grundlehren der Math., 127, Springer, Berlin, 1975.

[33] Y. Talebi and N. Vanaja, The torsion theory cogenerated by M-small modules, Comm. Algebra 30 (2002), 1449-1460.

[34] M. L. Teply, Generalizations of the simple torsion class and the splitting properties, Canad. J. Math. 27 (1975), 1056-1074.

[35] B. Ungor, S. Halicioglu and A. Harmanci, Modules in which inverse images of some submodules are direct summands, Comm. Algebra 44 (2016), 1496-1513.

[36] B. Ungor, S. Halicioglu and A. Harmanci, A dual approach to the theory of inverse split modules, J. Algebra Appl. 17 (2018), 1850148 (17 pages).

[37] N. Vanaja, All finitely generated M-subgenerated modules are extending, Comm. Algebra 24 (1996), 543-572.

[38] M. Wang, Some studies on QcF-coalgebras, International Symposium on Ring Theory (Kyongju, 1999), Trends Math., Birkhäuser, Boston, 2001, pp. 393-399.

[39] Y. Wang, Strongly lifting modules and strongly dual Rickart modules, Front. Math. China 12 (2017), 219-229.

[40] R. Wisbauer, Foundations of module and ring theory, Gordon and Breach, Reading, 1991.

Faculty of Mathematics and Computer Science, Babeş-Bolyai University, Str. M. KogăLniceanu 1, 400084 Cluj-Napoca, Romania

E-mail address: crivei@math.ubbcluj.ro

Department of Mathematics, Hacettepe University, 06800 Beytepe, Ankara, Turkey

E-mail address: keskin@hacettepe.edu.tr

Centre Régional des Métiers de L'Education et de la Formation (CRMef)-Tanger, Avenue My Abdelaziz, Souani, B.P.:3117, Tangier 90000, Morocco

E-mail address: tribak12@yahoo.com 